MATHEMATICS OF COMPUTATION

Volume 73, Number 247, Pages 1297-1324

S 0025-5718(03)01590-4

Article electronically published on July 29, 2003

\title{
DATA-SPARSE APPROXIMATION TO THE OPERATOR-VALUED FUNCTIONS OF ELLIPTIC OPERATOR
}

\author{
IVAN P. GAVRILYUK, WOLFGANG HACKBUSCH, AND BORIS N. KHOROMSKIJ
}

\begin{abstract}
In previous papers the arithmetic of hierarchical matrices has been described, which allows us to compute the inverse, for instance, of finite element stiffness matrices discretising an elliptic operator $\mathcal{L}$. The required computing time is up to logarithmic factors linear in the dimension of the matrix. In particular, this technique can be used for the computation of the discrete analogue of a resolvent $(z I-\mathcal{L})^{-1}, z \in \mathbb{C}$.

In the present paper, we consider various operator functions, the operator exponential $e^{-t \mathcal{L}}$, negative fractional powers $\mathcal{L}^{-\alpha}$, the cosine operator function $\cos (t \sqrt{\mathcal{L}}) \mathcal{L}^{-k}$ and, finally, the solution operator of the Lyapunov equation. Using the Dunford-Cauchy representation, we get integrals which can be discretised by a quadrature formula which involves the resolvents $\left(z_{k} I-\mathcal{L}\right)^{-1}$ mentioned above. We give error estimates which are partly exponentially, partly polynomially decreasing.
\end{abstract}

\section{INTRODUCTION}

This paper deals with the representation and approximation of operator-valued functions. In particular, we consider the four functions

$$
\begin{array}{rlrl}
\mathcal{F}_{1}(\mathcal{L}) & :=e^{-t \mathcal{L}} & \\
\mathcal{F}_{2}(\mathcal{L}) & :=\mathcal{L}^{-\alpha} & \text { for } \alpha>1, \\
\mathcal{F}_{3, k}(\mathcal{L}) & :=\cos (t \sqrt{\mathcal{L}}) \mathcal{L}^{-k} & & \text { for } k \in \mathbb{N}, \\
\mathcal{F}_{4}(\mathcal{L}) & :=\int_{0}^{\infty} e^{t \mathcal{L}^{\top} G e^{t \mathcal{L}} d t} &
\end{array}
$$

of an elliptic operator $\mathcal{L}$ representing four different types of problems. The functions $\mathcal{F}_{1}$ and $\mathcal{F}_{3, k}$ are of interest for parabolic (the operator exponential) and hyperbolic (the cosine operator) problems. However, for the practical application it is indispensable to realise (the application of) these operators in an efficient way.

Since we do not exclude finitely dimensional spaces, matrices are special examples of $\mathcal{L}$. In the matrix case, the most commonly used algorithms for approximating the action of the matrix exponential are based on Krylov subspace methods (see [20, 17]). The Dunford integral has previously been used in [23] (see also $\S 4.6$ of [22]) to achieve efficient inversions of differential operators, in particular, a method was derived and applied in 24] for solution of Burgers' equation. Although a different procedure was used in these references than those of the present paper, the above metioned results would be interesting for further comparisons of efficiency of the

Received by the editor July 9, 2002 and, in revised form, January 10, 2003.

2000 Mathematics Subject Classification. Primary 47A56, 65F30; Secondary 15A24, 15A99.

Key words and phrases. Operator-valued function, elliptic operator, $\mathcal{H}$-matrices. 
methods. Methods with a polynomial convergence based on a Dunford-Cauchy integral for symmetric and positive definite operators are proposed in [21]. Concerning the second order evolution problems and the cosine operator, new discretisation approaches have been recently proposed in [6] in the framework of strongly P-positive operators.

The purpose of this paper is to approximate the operator function in a first step by a sum

$$
\sum_{k=-N}^{N} \gamma_{k}(t)\left(z_{k} I-\mathcal{L}\right)^{-1}
$$

involving $2 N+1$ resolvents $\left(z_{k} I-\mathcal{L}\right)^{-1}$ (see (3.25) $)$. It is important to notice that the possible dependence on $t$ (in the case of $\mathcal{F}_{1}$ and $\mathcal{F}_{3, k}$ ) is described by the scalar coefficients $\gamma_{k}(t)$ only, while the values $z_{k}$ in (1.1) are independent of $t$.

Of course, the representation (1.1) is only semi-discrete, since $\mathcal{L}$ may still belong to an infinite-dimensional Banach space. In that case, $\mathcal{L}$ is to be replaced by a discretisation (say Galerkin matrix $\mathcal{L}_{h}$ ). The matrix resolvent $\left(z_{k} I-\mathcal{L}_{h}\right)^{-1}$ can be computed by the approximate inversion procedure offered by the hierarchical matrix technique (see [14], 15], [16], [4]). Here, we make use of the fact that $\mathcal{L}$ is assumed to be an elliptic operator and that the paper [2] ensures that $\left(z_{k} I-\mathcal{L}_{h}\right)^{-1}$ has the required hierarchical matrix property. The cost for the approximate inversion amounts to $O\left(n \log ^{q} n\right)$ for an $n \times n$ matrix $\mathcal{L}_{h}$. Note that in the special case of constant coefficients the direct approximation to the elliptic inverse by hierarchical matrices has been addressed in [18.

In order to reach the representation (1.1), we apply in $\$ 2$ the Dunford-Cauchy representation $]$ of the operator-valued function. The arising integral is discretised by means of the Sinc-quadrature. In the case of the operator exponential $\left(\mathcal{F}_{1}\right)$ one gets an expression (1.1) which converges exponentially as $N \rightarrow \infty$. The exponential convergence makes it possible to obtain an accurate approximation with $N$ being rather small.

Similarly, we can show in 44 that the corresponding quadrature result for $\mathcal{F}_{2}(\mathcal{L})=$ $\mathcal{L}^{-\alpha}$ is exponentially convergent, too.

The approximation of the cosine operator $\mathcal{F}_{3}$ in $\sqrt{6}$ turns out to be harder. Here, the approximation of the form (1.1) converges polynomially depending on the smoothness of the function the operator is applied to.

Since the cost for the matrix exponential $\mathcal{F}_{1}$ is only $O\left(n \log ^{q} n\right)$, the available exponential may be used for further applications. In $\$ 5$ we consider $\mathcal{F}_{4}(\mathcal{L})=$ $\int_{0}^{\infty} e^{t \mathcal{L}^{\top}} G e^{t \mathcal{L}} d t$, which represents the solution to the Lyanunov equation. After a Sinc-quadrature applied to the integral over $[0, \infty)$, we obtain an exponentially convergent quadrature rule involving the operator $e^{t_{k} \mathcal{L}^{\top}} G e^{t_{k} \mathcal{L}}$. Using the approximations to $\mathcal{F}_{1}$, we reach again a representation (1.1).

\section{INTEGRAL REPRESENTATION TO OPERATOR-VALUED FUNCTIONS}

Before we describe the integral representation in $₫ 2.2$, we formulate a condition ensuring a fast convergent quadrature.

\footnotetext{
${ }^{1}$ We remark that another approach can be based on the Cayley transform (cf. [6], [7], 10]).
} 
2.1. Operators of $\left(f_{S}, f_{R}\right)$-type. Let $A: X \rightarrow X$ be a linear densely defined closed operator in $\mathrm{X}$ with the spectral set $s p(A)$. Given a curve $\Gamma$ enveloping the spectrum of $A$, the behaviour of the resolvent $(z I-\mathcal{L})^{-1}$ as a function of $z \in \Gamma$ contains important information about the operator $A$ and allows us to develop a calculus of functions of $A$ (cf. [6, 8, 9, 12]). Strongly P-positive operators were introduced in [6] and play an important role in the theory of second order difference equations, evolution differential equations as well as the cosine operator family (see 6]). Using this class of operators, the solution operators for parabolic (the operator exponential) and elliptic (the normalised hyperbolic sine operator family) equations are introduced, studied and sparsely approximated in [8, 9]. These discretisations have the remarkable property that their convergence rate is exponential. Due to the application of $\mathcal{H}$-matrices to represent the elliptic resolvents, we arrive at a linear-logarithmic cost of the resultant discrete representations.

An exponentially convergent discretisation for the cosine operator generated by a strongly P-positive operator remains to be an open question. In this paper, we restrict ourselves to a class of operators of $\left(f_{S}, f_{R}\right)$-type to be defined below. In that case, we are able to construct polynomially convergent approximations.

Let $\Gamma_{S}$ be a curve in the complex plane $z=\xi+i \eta$ defined by the equation $\xi=f_{S}(\eta)$ in the coordinates $\xi, \eta$. We denote by

$$
\Omega_{\Gamma_{S}}:=\left\{z=\xi+i \eta: \xi>f_{S}(\eta)\right\}
$$

the domain inside of $\Gamma_{S}$. In what follows, we suppose that this curve lies in the right half-plane of the complex plane and contains $s p(A)$, i.e., $s p(A) \subset \mathbb{C} \backslash \Omega_{\Gamma_{S}}$. Now, we are in the position to give the following definition.

Definition 2.1. Given an operator $A: X \rightarrow X$, let $f_{S}(\eta)$ and $f_{R}(z)$ be functions such that

$$
\left\|(z I-A)^{-1}\right\|_{X \rightarrow X} \leq f_{R}(z) \quad \text { for all } z \in \mathbb{C} \backslash \Omega_{\Gamma_{S}} .
$$

Note that $\Gamma_{S}$ is defined by means of $f_{S}$ (cf. (2.10). Then we say that the operator $A: X \rightarrow X$ is of $\left(f_{S}, f_{R}\right)$-type.

Note that a strongly P-positive operator (defined in [6]) is also an operator of $\left(f_{S}, f_{R}\right)$-type with the special choice

$$
f_{S}(\eta)=a \eta^{2}+\gamma_{0}, \quad f_{R}(z)=M /(1+\sqrt{|z|}), \quad a>0, \quad \gamma_{0}>0, \quad M>0 .
$$

In particular, strongly elliptic partial differential operators are strongly P-positive.

In order to get exponentially convergent discretisations, we are interested in operators of $\left(f_{S}, f_{R}\right)$-type with an exponentially increasing function $f_{S}$. Some examples of such operators are given in the appendix.

2.2. Integral representation to operators of $\left(f_{S}, f_{R}\right)$-type. Let $\mathcal{L}$ be a linear, densely defined, closed operator of $\left(f_{S}, f_{R}\right)$-type in a Banach space $X$. We choose an integration curve $\Gamma_{I}:=\left\{z=f_{I}(\eta)+i \eta\right\}$ enveloping the so-called "spectral curve" $\xi=f_{S}(\eta)$ (see Figure 1). Let $F(z)$ be a complex-valued function that is analytic inside of the integration curve $\Gamma_{I}$. The next simple theorem offers conditions under which one can define a bounded operator $F(\mathcal{L})$. 


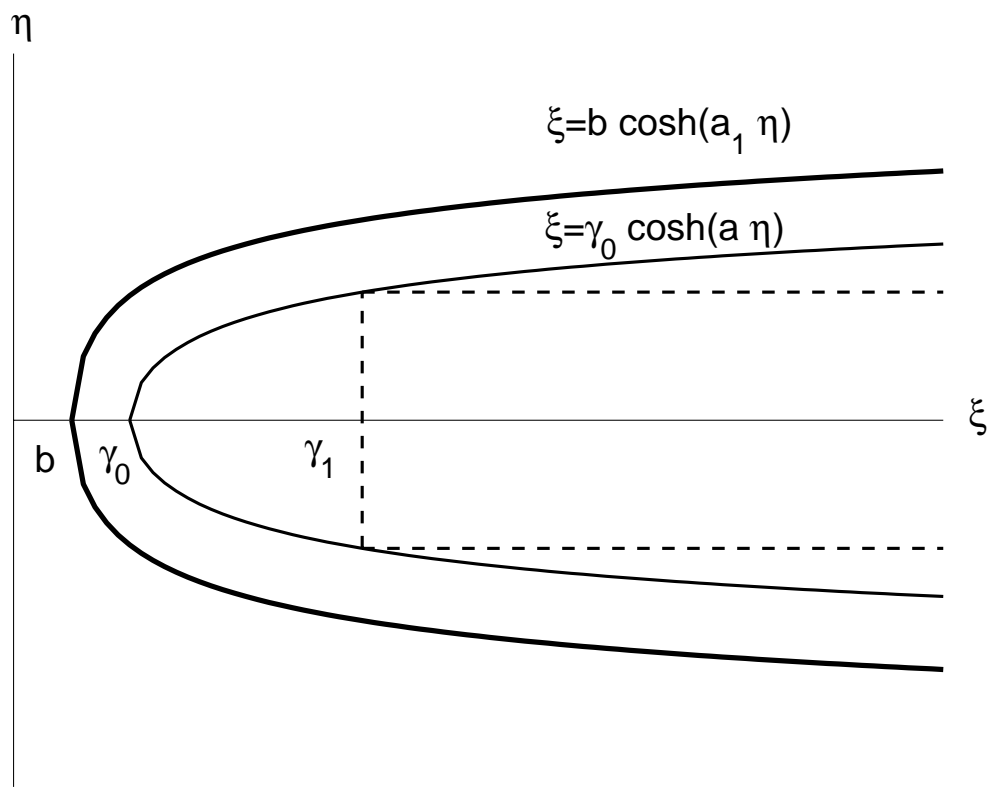

Figure 1. The spectral curve $\Gamma_{S}$ and the integration curve $\Gamma_{I}$.

Theorem 2.2. Let $\xi=f_{I}(\eta)$ be an even function and assume that the improper integral

$$
\int_{-\infty}^{\infty}\left|\Phi_{1}(\eta)\right| f_{R}\left(f_{I}(\eta)-i \eta\right) d \eta \quad \text { with } \Phi_{1}(\eta)=F\left(f_{I}(\eta)-i \eta\right)\left[f_{I}^{\prime}(\eta)-i\right]
$$

converges. Then the Dunford-Cauchy integral

$$
F(\mathcal{L})=\frac{1}{2 \pi i} \int_{\Gamma_{I}} F(z)(z I-\mathcal{L})^{-1} d z=-\frac{1}{2 \pi i} \int_{-\infty}^{\infty} \Phi_{1}(\eta)\left[\left(f_{I}(\eta)-i \eta\right) I-\mathcal{L}\right]^{-1} d \eta
$$

defines a bounded operator $F(\mathcal{L})$.

Proof. Using the Dunford-Cauchy integral, we represent $F(\mathcal{L})$ by

$$
\frac{1}{2 \pi i} \int_{\Gamma_{I}} F(z)(z I-\mathcal{L})^{-1} d z
$$

After the parametrisation $z=f_{I}(\eta)+i \eta, \eta \in(-\infty, \infty)$, we get

$$
F(\mathcal{L})=\frac{1}{2 \pi i} \int_{-\infty}^{\infty} \Phi(\eta ; \mathcal{L}) d \eta
$$

with $\Phi(\eta ; \mathcal{L})=\Phi_{1}(\eta)\left[f_{I}(\eta)-i \eta-\mathcal{L}\right]^{-1}, \Phi_{1}(\eta)=F\left(f_{I}(\eta)-i \eta\right)\left[f_{I}^{\prime}(\eta)-i\right]$. Due to the fact that $\mathcal{L}$ is an operator of $\left(f_{S}, f_{R}\right)$-type, we further get the estimate

$$
\|F(\mathcal{L})\| \leq \int_{-\infty}^{\infty}\left|\Phi_{1}(\eta)\right| f_{R}\left(f_{I}(\eta)-i \eta\right) d \eta<\infty,
$$

which yields the existence of the bounded operator $F(\mathcal{L})$. 


\section{Approximation to the operator exponential}

In $\S \$ 3.2$ 3.4 we consider the Sinc-quadrature based on the representation (2.5). Finally in $\S 3.53 .6$ we apply this approach to the operator exponential. Because of the new integration path, the result is different from a previous one used in [8] and recalled in $\$ 3.1$.

The quadrature result for the exponential will be used later in $\{5$, where we consider the solution operator $\mathcal{F}_{4}(\mathcal{L})=\int_{0}^{\infty} e^{t \mathcal{L}^{\top}} G e^{t \mathcal{L}} d t$ to the Lyapunov equation.

3.1. Previous approach. Exponentially convergent quadrature rules for the function $F_{1}$ generated by a strongly P-positive elliptic operator are analysed in 8 . In fact, for an integration parabola

$$
\Gamma_{b(k)}=\left\{z=f_{I}(\eta)+i \eta: \eta \in(-\infty, \infty)\right\}, \quad f_{I}(\eta):=\tilde{a} \eta^{2}+b(k),
$$

with given $k>1, \tilde{a}=a / k, b(k)=\gamma_{0}-(k-1) /(4 a), k>1$, the quadrature

$$
\exp _{L}(-\mathcal{L}):=\sum_{p=-L}^{L} c_{p} e^{-z_{p}}\left(z_{p} I-\mathcal{L}\right)^{-1}
$$

with the parameters $z_{p}=f_{I}(p h)+i p h, h=\sqrt[3]{2 \pi d k /\left(L^{2} a\right)}, d=(1-1 / \sqrt{k}) k /(2 a)$ involves $2 L+1$ terms. For $c_{p}$ see [8, Algorithm 2.5]. It yields an approximation which converges exponentially as $L \rightarrow \infty$ :

$$
\left\|\exp (-\mathcal{L})-\exp _{L}(-\mathcal{L})\right\| \leq C\left[\frac{2 \sqrt{k} \exp \left(-L^{2 / 3}\right)}{\sqrt{a t}\left(1-\exp \left(-s L^{2 / 3}\right)\right)}+\frac{k \exp \left(-t s L^{2 / 3}\right)}{\sqrt{\pi} t \sqrt[3]{2 \pi d k a^{2} L}}\right],
$$

where $s=\sqrt[3]{(2 \pi d)^{2} a / k}$ and $C$ is a positive constant.

Based on (3.1), an efficient parallel algorithm of almost linear complexity to compute the matrix exponential has been proposed and analysed in [8. Since in the present paper we deal with a more restricted class of operators, we introduce a better algorithm based on Lemma 3.2 below.

3.2. A new representation of the operator exponential. Let $\mathcal{L}$ be a linear, densely defined, closed operator of $\left(f_{S}, f_{R}\right)$-type in a Banach space $X$, where

$$
f_{S}(\eta)=\gamma_{0} \cosh (a \eta) \quad \text { with } \gamma_{0}>0, \quad f_{R}(z)=1 /|\Im m z| \quad \text { if } \Re e z>\gamma_{0} .
$$

The function $f_{S}(\eta)$ corresponds to the spectral curve

$$
\Gamma_{S}=\left\{z=\xi+i \eta: \xi=\gamma_{0} \cosh (a \eta)\right\}
$$

containing the spectrum $s p(\mathcal{L})$ of the operator $\mathcal{L}$.

Definition 3.1. The class of $\left(f_{S}, f_{R}\right)$-type operators specified by $f_{S}, f_{R}$ from (3.2) is denoted by $\mathbf{E}_{S, R}$.

For the sake of simplicity, in what follows, we consider operators with real spectrat that are bounded from below by $\gamma_{0}$ (cf. (3.2)).

In the following, we use the infinite strip

$$
D_{d}:=\{z \in \mathbb{C}:-\infty<\Re e z<\infty,|\Im m z|<d\}
$$

as well as the finite rectangles $D_{d}(\epsilon)$ defined for $0<\epsilon<1$ by

$$
D_{d}(\epsilon)=\{z \in \mathbb{C}:|\Re e z|<1 / \epsilon,|\Im m z|<d(1-\epsilon)\} .
$$

\footnotetext{
${ }^{2}$ Analogously, one can consider operators with spectra in a half-strip $S_{\mu}$ (see [5.5) where the resolvent is bounded by $f_{R}(z)=\operatorname{dist}^{-1}\left(z, S_{\mu}\right), z \in \mathbb{C} \backslash \overline{S_{\mu}}$.
} 


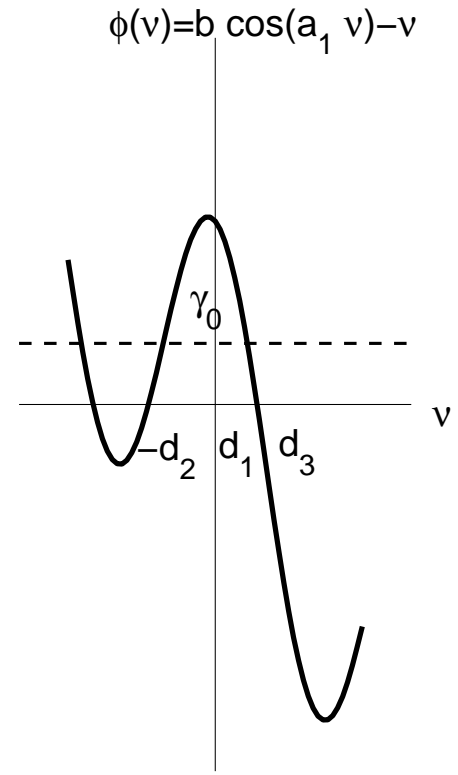

a): $b=3.0 ; a_{1}=1.5$

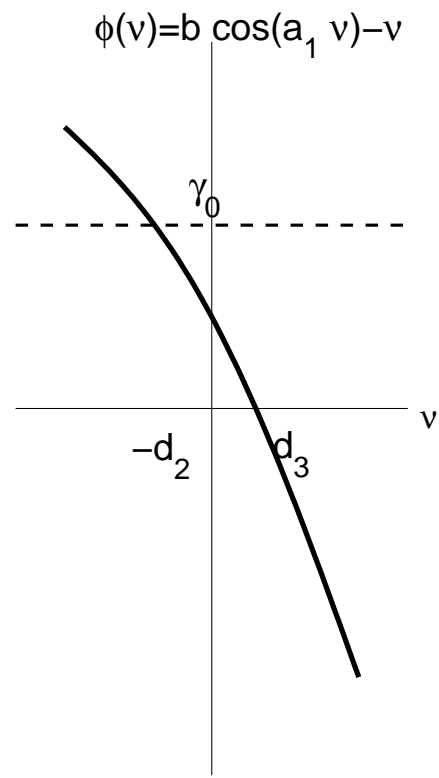

b): $b=1.0 ; a_{1}=0.5$

Figure 2. Calculation of the width of the analyticity strip.

For $1 \leq p \leq \infty$, introduce the family $\mathbf{H}^{p}\left(D_{d}\right)$ of all operator-valued functions which are analytic in $D_{d}$, such that for each $\mathcal{F} \in \mathbf{H}^{p}\left(D_{d}\right)$ it holds that $\|\mathcal{F}\|_{\mathbf{H}^{p}\left(D_{d}\right)}<$ $\infty$ with

$$
\|\mathcal{F}\|_{\mathbf{H}^{p}\left(D_{d}\right)}:= \begin{cases}\lim _{\epsilon \rightarrow 0}\left(\int_{\partial D_{d}(\epsilon)}\|\mathcal{F}(z)\|^{p}|d z|\right)^{1 / p} & \text { if } 1 \leq p<\infty \\ \lim _{\epsilon \rightarrow 0} \sup _{z \in D_{d}(\epsilon)}\|\mathcal{F}(z)\| & \text { if } p=\infty\end{cases}
$$

In the following, we consider an integral representation for $e^{-t \mathcal{L}}$ with $\mathcal{L} \in \mathbf{E}_{S, R}$, where the integrand is proved to be in the class $\mathbf{H}^{p}\left(D_{d}\right)$. The proper choice of the parameter $d>0$ will be determined in the sequel. We choose a parameter $b \in\left(0, \gamma_{0}\right)$, where $\gamma_{0}$ is defined in $(\underline{3.2})$, and we consider the equation

$$
\varphi(\nu):=b \cos (a \nu)-\nu=\gamma_{0}, \quad 0<b<\gamma_{0}, a>0 .
$$

Let $d_{1}$ be the minimal positive solution (if existing), and let $-d_{2}$ be the maximal negative solution $\left(d_{2}>0\right)$. Then $d=\min \left\{d_{1}, d_{2}\right\}$ is the desired parameter (see Figure 2).

Lemma 3.2. Let $\mathcal{L}$ be an operator of the class $\mathbf{E}_{S, R}$. Choose a curve (integration curve) $\Gamma_{I}=\left\{z=\xi+i \eta: \xi=b \cosh \left(a_{1} \eta\right)\right\}$ with $a_{1}<a, b \in\left(0, \gamma_{0}\right)$. Then the operator exponential $I(t ; \mathcal{L})=e^{-t \mathcal{L}}$ can be represented by the Dunford-Cauchy integral

$$
I(t ; \mathcal{L})=\frac{1}{2 \pi i} \int_{\Gamma_{I}} e^{-z t}(z I-\mathcal{L})^{-1} d z=-\frac{1}{2 \pi i} \int_{-\infty}^{\infty} F_{1}(\eta, t) d \eta
$$


(cf. [5]), where the integrand

$$
F_{1}(\eta, t)=e^{-z t}\left(b a_{1} \cosh \left(a_{1} \eta\right)+i\right)(z I-\mathcal{L})^{-1} \quad \text { with } z=b \cosh \left(a_{1} \eta\right)+i \eta, \eta \in \mathbb{R},
$$

can be estimated on the real axis by

$$
\left\|F_{1}(\eta, t)\right\| \leq C b a_{1}(1+|\eta|)^{-1} e^{a_{1}|\eta|-b t \cosh \left(a_{1} \eta\right)} \quad \text { for } \eta \in \mathbb{R} .
$$

Moreover, $F_{1}(\cdot, t)$ can be analytically extended into the strip $D_{d}$ of the width $d>0$ constructed above and it belongs to the class $\mathbf{H}^{p}\left(D_{d}\right)$ for all $p \in[1, \infty]$.

Proof. Since the operator $\mathcal{L}$ is of $\left(f_{S}, f_{R}\right)$-type with $f_{S}, f_{R}$ given by (3.2), we can choose

$$
f_{I}(\eta)=b \cosh \left(a_{1} \eta\right), \quad a_{1}<a, b \in\left(0, \gamma_{0}\right) .
$$

After parametrisation of the path $\Gamma_{I}$ we get

$$
I(t):=\frac{1}{2 \pi i} \int_{\Gamma_{I}} e^{-z t}(z I-\mathcal{L})^{-1} d z=\frac{1}{2 \pi i} \int_{-\infty}^{\infty} F_{1}(\eta, t) d \eta
$$

with

$$
F_{1}(\eta, t)=F_{11}(\eta, t)(z I-\mathcal{L})^{-1} \quad \text { and } \quad\left\{\begin{array}{l}
F_{11}(\eta, t)=e^{-z t}\left(b a_{1} \sinh \left(a_{1} \eta\right)+i\right), \\
z=b \cosh \left(a_{1} \eta\right)+i \eta
\end{array}\right.
$$

Since the operator $\mathcal{L}$ is of $\left(f_{S}, f_{R}\right)$-type with $f_{S}, f_{R}$ given by (3.2), we can choose the integration path in accordance with (3.9) and get for $|\eta| \rightarrow \infty$

$$
\left|\Phi_{1}(\eta)\right|=\left|F_{11}\left(f_{I}(\eta)-i \eta\right)\left[f_{I}^{\prime}(\eta)-i\right]\right| \leq C \exp \left(a_{1}|\eta|-b t \cosh \left(a_{1} \eta\right)\right) .
$$

Furthermore, there exists a constant $M_{F_{1}}$ such that

$$
\left|f_{R}\left(f_{I}(\eta)-i \eta\right)\right| \leq M_{F_{1}} /(1+|\eta|)
$$

Thus, all assumptions of Theorem 2.2 are fulfilled and the integral (3.10) converges for all $t>0$.

Replacing in $F_{1}(\eta, t)$ the real variable $\eta$ by $z=\eta+i \nu,|\nu|<d$ and using the estimate for the resolvent, we get in the strip $D_{d}=\{z=(\eta, \nu): \eta \in(-\infty, \infty)$, $|\nu|<d\}$

$$
\begin{aligned}
F_{1}(\eta+i \nu, t)= & \mathcal{O}\left((1+|\eta|)^{-1} \exp \left(-b t e^{a_{1}|\eta|} \cos \left(a_{1} \nu\right)+b a_{1}|\eta|\right)\right) \\
& +i \mathcal{O}\left((1+\eta)^{-1} \exp \left(-b t e^{a_{1}|\eta|} \cos \left(a_{1} \nu\right)+a_{1} \eta\right)\right)
\end{aligned}
$$

Analyticity of the integrand can be violated only if $b \cosh \left(a_{1}(\eta+i \nu)\right)-\nu+i \eta=0$ or if the set

$$
\begin{aligned}
Z & =\left\{b \cosh \left(a_{1}(\eta+i \nu)\right)-\nu+i \eta: \eta \in(-\infty, \infty),|\nu|<d\right\} \\
=\left\{b \cos \left(a_{1} \nu\right) \cosh \left(a_{1} \eta\right)-\nu+i\left(b \sin \left(a_{1} \nu\right) \sinh \left(a_{1} \eta\right)+\eta\right)\right. & \quad: \eta \in(-\infty, \infty),|\nu|<d\}
\end{aligned}
$$

intersects the part of the real axis $\eta>\gamma_{0}$ where the spectrum of $\mathcal{L}$ is situated (in this case the resolvent is unbounded). The intersection of $Z$ with the real axis is $\{z \in Z: \Im m z=0\}=\left\{b \cos \left(a_{1} \nu\right)-\nu:|\nu|<d\right\}$. Now, we need the following condition to be valid:

$$
\varphi(\nu)=b \cos \left(a_{1} \nu\right)-\nu<\gamma_{0}
$$

Let $d_{1},-d_{2}$ be defined as the solutions of equation (3.6) as above. Then the width of the strip in which $F_{1}$ can be extended analytically is $d=\min \left\{d_{1}, d_{2}\right\}$ (see also 
Figure 2). It follows from (3.13) that the integrand belongs to the class $\mathbf{H}^{p}\left(D_{d}\right)$ for all $p \in[0, \infty)$.

3.3. The Sinc-quadrature. Following [22, 12, 8, we construct a quadrature rule for the integral in (3.7) by using the Sinc approximation. Let

$$
S(k, h)(x)=\frac{\sin [\pi(x-k h) / h]}{\pi(x-k h) / h} \quad(k \in \mathbb{Z}, h>0, x \in \mathbb{R})
$$

be the $k$ th Sinc function with step size $h$, evaluated at $x$. Given $f \in \mathbf{H}^{p}\left(D_{d}\right), h>0$, and $N \in \mathbb{N}$, we use the notation

$$
\begin{array}{rlrl}
I(f) & =\int_{\mathbb{R}} f(\xi) d \xi & & \\
T(f, h) & =h \sum_{k=-\infty}^{\infty} f(k h), & T_{N}(f, h)=h \sum_{k=-N}^{N} f(k h), \\
C(f, h) & =\sum_{k=-\infty}^{\infty} f(k h) S(k, h), & E(f, h) & =f-C(f, h), \\
\eta(f, h) & =I(f)-T(f, h), & \eta_{N}(f, h) & =I(f)-T_{N}(f, h)
\end{array}
$$

( $I$ : integral, $T$ : trapezoidal rule, $C$ : cardinal series representation, $E$ : its error, $\eta, \eta_{N}$ : quadrature errors). Note that $C(f, h)$ is a function of $x \in \mathbb{R}$. Furthermore, we need the notation of one-sided limits:

$$
f\left(\xi \pm i d^{-}\right)=\lim _{\delta \rightarrow d ; \delta<d} f(\xi \pm i \delta) \quad \text { for } \xi, d \in \mathbb{R} .
$$

3.4. Convergence analysis. Adapting the ideas of [22, 12, 8, one can prove the following approximation results for functions from $\mathbf{H}^{1}\left(D_{d}\right)$, describing the accuracy of $T(f, h)$ and $T_{N}(f, h)$.

Lemma 3.3. For any operator-valued function $f \in \mathbf{H}^{1}\left(D_{d}\right)$, it holds that

$$
\eta(f, h)=\frac{i}{2} \int_{\mathbb{R}}\left\{\frac{f\left(\xi-i d^{-}\right) e^{-\pi(d+i \xi) / h}}{\sin [\pi(\xi-i d) / h]}-\frac{f\left(\xi+i d^{-}\right) e^{-\pi(d-i \xi) / h}}{\sin [\pi(\xi+d) / h]}\right\} d \xi
$$

providing the estimate

$$
\|\eta(f, h)\| \leq \frac{e^{-\pi d / h}}{2 \sinh (\pi d / h)}\|f\|_{\mathbf{H}^{1}\left(D_{d}\right)} .
$$

If, in addition, $f$ satisfies on $\mathbb{R}$ the condition

$$
\|f(\xi)\|<c(1+|\xi|)^{-1} \exp \left(a|\xi|-b e^{a|\xi|}\right), \quad a, b, c>0,
$$

then

$$
\left\|\eta_{N}(f, h)\right\| \leq \frac{2 c}{a b}\left[\frac{e^{-b} e^{-2 \pi d / h}}{1-e^{-2 \pi d / h}}+\frac{2}{1+N h} \exp \left(-b e^{a h N}\right)\right]
$$

with the constant d from $\mathbf{H}^{1}\left(D_{d}\right)$.

Proof. Let $E(f, h)$ be the error from (3.16). Analogously to [22, Thm. 3.1.2], $E(f, h)(z)$ equals

$$
\frac{\sin (\pi z / h)}{2 \pi i} \int_{\mathbb{R}}\left\{\frac{f(\xi-i d)}{(\xi-z-i d) \sin [\pi(\xi-i d) / h]}-\frac{f(\xi+i d)}{(\xi-z+i d) \sin [\pi(\xi+i d) / h]}\right\} d \xi
$$


and after replacing $z$ by $x$, we have

$$
\eta(f, h)=\int_{\mathbb{R}} E(f, h)(x) d x
$$

because of $\int_{\mathbb{R}} S(k, h)(x) d x=h$. After interchanging the order of integration and using

$$
\frac{1}{2 \pi i} \int_{\mathbb{R}} \frac{\sin (\pi x / h)}{ \pm(\xi-x)-i d} d x=\frac{i}{2} e^{-\pi(d \pm i \xi) / h},
$$

we obtain (3.17). Using the estimate $\sinh (\pi d / h) \leq|\sin [\pi(\xi \pm i d) / h]| \leq \cosh (\pi d / h)$ (see [22, p. 133]), the assumption $f \in \mathbf{H}^{1}\left(D_{d}\right)$ and identity (3.17), we obtain the desired bound (3.18). The assumption (3.19) now implies

$$
\begin{aligned}
\left\|\eta_{N}(f, h)\right\| & \leq\|\eta(f, h)\|+h \sum_{|k|>N}\|f(k h)\| \\
(3.21) \leq & \leq \frac{\exp (-\pi d / h)}{2 \sinh (\pi d / h)}\|f\|_{\mathbf{H}^{1}\left(D_{d}\right)}+c h \sum_{k:|k|>N}(1+k h)^{-1} \exp \left(a|k h|-b e^{a|k h|}\right) .
\end{aligned}
$$

For the last sum we use the simple estimate

$$
\begin{aligned}
& \sum_{k:|k|>N}(1+|k h|)^{-1} \exp \left(a|k h|-b e^{a|k h|}\right) \\
& \quad=2 \sum_{k=N+1}^{\infty}(1+k h)^{-1} \exp \left(a|k h|-b e^{a|k h|}\right) \\
& \quad \leq \frac{2}{1+N h} \int_{N}^{\infty} \exp \left(a|k h|-b e^{a|k h|}\right) d x=\frac{2}{a b h(1+N h)} \exp \left(-b e^{a h N}\right) .
\end{aligned}
$$

It follows from (3.19) that

$$
\|f\|_{\mathbf{H}^{1}\left(D_{d}\right)} \leq 2 c \int_{-\infty}^{\infty} e^{a|x|} \exp \left(-b e^{a|x|}\right) d x \leq \frac{4 c}{a b} e^{-b},
$$

which together with (3.21) and (3.22) implies

$$
\left\|\eta_{N}(f, h)\right\| \leq \frac{2 c}{a b}\left[\frac{\exp (-b-\pi d / h)}{\sinh (\pi d / h)}+\frac{1}{(1+N h)} \exp \left(-b e^{a h N}\right)\right],
$$

completing the proof.

3.5. Application to the exponential. The operator exponential $I(t ; \mathcal{L})=e^{-t \mathcal{L}}$ is represented as the integral according to Lemma 3.2. Applying the quadrature rule $T_{N}$ to the operator-valued function $f(\eta):=-\frac{h}{2 \pi i} F_{1}(\eta, t)$, where $F_{1}(\eta, t)$ is given by (3.8), we obtain for the operator family $\{I(t) \equiv I(t ; \mathcal{L}): t>0\}$ (cf. (3.14)) that

$$
I(t) \approx T_{N}(t) \equiv T_{N}(f, h)=-\frac{h}{2 \pi i} \sum_{k=-N}^{N} F_{1}(k h, t) .
$$

The error analysis is given by the following theorem. 
Theorem 3.4. Given the spectral curve $\Gamma_{S}$ from (3.3) associated with $f_{S}(\eta)=$ $\gamma_{0} \cosh (a \eta)$, choose the integration curve $\left\{z=f_{I}(\eta)+i \eta: \eta \in \mathbb{R}\right\}$ with $f_{I}(\eta):=$ $\left.b \cosh \left(a_{1} \eta\right), b \in\left(0, \gamma_{0}\right), a_{1}<a\right\}$ and set $h=\frac{\log N}{a_{1} N}$. Then

$$
\left\|I(t)-T_{N}(t)\right\| \leq C \frac{1}{t}\left(e^{-b t-2 \pi d N / \log N}+\frac{e^{-b t N}}{1+(\log N) / a_{1}}\right) .
$$

Proof. Substituting in (3.20) $F_{1}$ for $f, a_{1}$ for $a, b t$ for $b$ and specifying $h=\frac{\log N}{a_{1} N}$, we conclude (3.24) from $\left\|\eta_{N}\left(F_{1}, h\right)\right\| \leq C \frac{1}{t}\left(e^{-b t-2 \pi d N / \log N}+\frac{e^{-b t N}}{1+(\log N) / a_{1}}\right)$.

3.6. The computational scheme. The exponential convergence of our quadrature rule allows us to introduce the following algorithm for the approximation of the operator exponential with a given tolerance $\varepsilon>0$. Note that the time-variable $t \in(0, \infty)$ enters only the coefficients of our quadrature rule, while all resolvents appear to be independent on $t$.

Proposition 3.5. (a) Let $\varepsilon>0$ be given. In order to obtain $\left\|I(t)-T_{N}(t)\right\| \leq C \frac{\varepsilon}{t}$ uniformly with respect to $t>0$, choose

$$
\begin{aligned}
a_{1} & >a, \quad b \in\left(0, \gamma_{0}\right), \quad N=O(|\log \varepsilon|), \quad h=\frac{\log N}{a_{1} N}, \\
z_{k} & =f_{I}(k h)+i k h \quad(k=-N, \ldots, N) \quad \text { with } f_{I}(\eta)=b \cosh \left(a_{1} \eta\right), \\
\gamma_{k}(t) & =e^{-z_{k} t} \frac{h}{2 \pi i}\left(b a_{1} \cosh \left(a_{1} k h\right)+i\right) .
\end{aligned}
$$

Then $T_{N}(t)$ is a linear combination of $2 N+1$ resolvents with scalar weights depending on $t$ :

$$
T_{N}(t)=\sum_{k=-N}^{N} \gamma_{k}(t)\left(z_{k} I-\mathcal{L}\right)^{-1},
$$

so that the computation of $T_{N}(t)$ requires $2 N+1=O(|\log \varepsilon|)$ evaluations of the resolvents $\left(z_{k} I-\mathcal{L}\right)^{-1}, k=-N, \ldots, N$.

(b) The evaluations (or approximations) of the resolvents can be performed in parallel. Note that the shifts $z_{k}$ are independent of $t$.

(c) Having evaluated the resolvents, $T_{N}(t)$ can be determined in parallel for different $t$-values $t_{1}, t_{2}, \ldots$.

Proof. Use (3.24) for the error estimate. Then (3.23) and (3.11) show that $\gamma_{k}(t)=$ $-\frac{h}{2 \pi i} F_{11}(k h, t)$.

Remark 3.6. It is also possible to ensure $\left\|I(t)-T_{N}(t)\right\| \leq C \varepsilon$ for $t>0$ by the choice $N=O(|\log \varepsilon|-\log t)$. Then, however, the quadrature points $z_{k}$ become $t$-dependent.

\section{The OPERATOR $\mathcal{L}^{-\alpha}, \alpha>1$}

In this section we consider the operator family $\mathcal{L}^{-\alpha}, \alpha>1$ (cf. [7]). The practical significance of this class of operators is related, in particular, to the operators $\mathcal{L}^{ \pm 1 / 2}$, which play an important role in the analysis of elliptic Poincaré-Steklov operators [9] including preconditioning issues. In particular, the factorised representation $\mathcal{L}^{\alpha}=\mathcal{L} \mathcal{L}^{\alpha-2} \mathcal{L}, 2-\alpha>1$, holds, indicating a constructive way to approximate positive powers of an elliptic operator. 
4.1. The integral representation. We begin this section with a representation theorem for negative powers of an operator of $\left(f_{S}, f_{R}\right)$-type.

Lemma 4.1. Let $\mathcal{L}$ be an operator from the class $\mathbf{E}_{S, R}$. Choose a curve (the integration curve) $\Gamma_{I}=\left\{z=\xi+i \eta: \xi=b \cosh \left(a_{1} \eta\right)\right\}$ with $a>0, b \in\left(0, \gamma_{0}\right)$. Then the operator $\mathcal{L}^{-\alpha}, \alpha>1$, can be represented by the Dunford-Cauchy integral

$$
\mathcal{L}^{-\alpha}=\frac{1}{2 \pi i} \int_{\Gamma_{I}} z^{-\alpha}(z I-\mathcal{L})^{-1} d z=-\frac{1}{2 \pi i} \int_{-\infty}^{\infty} F_{2}(\eta, t) d \eta,
$$

where the integrand

$$
F_{2}(\eta, t)=z^{-\alpha}\left(b a_{1} \sinh \left(a_{1} \eta\right)+i\right)(z-\mathcal{L})^{-1}, \quad z=b \cosh \left(a_{1} \eta\right)+i \eta, \eta \in \mathbb{R}
$$

can be estimated on the real axis by

$$
\left\|F_{2}(\eta, t)\right\| \leq C a_{1} b^{1-\alpha}(1+|\eta|)^{-1} e^{-a_{1}(\alpha-1)|\eta|} .
$$

Moreover, $F_{2}(\cdot, t)$ can be analytically extended into a strip $D_{d}$ of width $d>0$ (defined as in the proof of Lemma 3.2) and it belongs to the class $\mathbf{H}^{p}\left(D_{d}\right)$ for all $p \in[1, \infty]$.

Proof. For the sake of simplicity we consider again the case when the spectrum of $\mathcal{L}$ is situated on the real axis. After parametrisation of the path $\Gamma_{I}$, we get

$$
\begin{aligned}
\mathcal{L}^{-\alpha} & =\frac{1}{2 \pi i} \int_{\Gamma_{I}} z^{-\alpha}(z I-\mathcal{L})^{-1} d z \\
& =\frac{1}{2 \pi i} \int_{\infty}^{0}\left(b \cosh \left(a_{1} \eta\right)+i \eta\right)^{-\alpha}\left(b a_{1} \sinh \left(a_{1} \eta\right)+i\right)\left(b \cosh \left(a_{1} \eta\right)+i \eta-\mathcal{L}\right)^{-1} d \eta \\
& +\frac{1}{2 \pi i} \int_{0}^{-\infty}\left(b \cosh \left(a_{1} \eta\right)+i \eta\right)^{-\alpha}\left(b a_{1} \sinh \left(a_{1} \eta\right)+i\right)\left(b \cosh \left(a_{1} \eta\right)+i \eta-\mathcal{L}\right)^{-1} d \eta \\
& =-\frac{1}{2 \pi i} \int_{-\infty}^{\infty} F_{2}(\eta, t) d \eta
\end{aligned}
$$

with $F_{2}(\eta, t)$ from (4.2). Since the operator $\mathcal{L}$ is from the class $\mathbf{E}_{S, R}$, it holds that

$$
\left\|(z I-\mathcal{L})^{-1}\right\| \leq \frac{1}{|\Im m z|}
$$

(cf. (3.2) ) and we can choose the integration path by means of

$$
f_{I}(\eta)=b \cosh \left(a_{1} \eta\right), \quad a_{1}<a, b \in\left(0, \gamma_{0}\right) .
$$

It is easy to see that for $F(z)=z^{-\alpha}, \alpha>1$, it holds that

$$
\left|\Phi_{1}(\eta)\right|=\left|F\left(f_{I}(\eta)-i \eta\right)\left[f_{I}^{\prime}(\eta)-i\right]\right| \leq C e^{-\alpha a_{1}|\eta|} \cdot e^{a_{1}|\eta|}=e^{-a_{1}(\alpha-1)|\eta|}
$$

(see (2.4) ). Moreover, there exists a constant $M_{f}$ such that

$$
\left|f_{R}\left(f_{I}(\eta)-i \eta\right)\right| \leq M_{f} /(1+|\eta|) \quad \text { for }|\eta| \rightarrow \infty .
$$

Since all assumptions of Theorem 2.2 are fulfilled, the Dunford-Cauchy integral converges and the estimate (4.3) holds. The width $d$ of the strip of analyticity of $F_{2}(\cdot, t)$ can be estimated as in Lemma 3.2 
4.2. Convergence analysis and computational scheme. Adapting the ideas of [22, 12, 8, one can prove the following approximation results for functions from $\mathbf{H}^{1}\left(D_{d}\right)$ (see also Lemma 3.3 above).

Lemma 4.2. Let $f \in \mathbf{H}^{1}\left(D_{d}\right)$ and let $f$ satisfy the condition

$$
\|f(\xi)\| \leq c(1+|\xi|)^{-1} \exp (-a|\xi|) \quad \text { for all } \xi \in \mathbb{R} \text { and some } a, c>0 \text {. }
$$

Then, taking $h=\sqrt{\frac{2 \pi d}{a N}}$, we obtain

$$
\left\|\eta_{N}(f, h)\right\| \leq \frac{2 c}{a}\left[\frac{2 e^{-2 \pi d / h}}{1-e^{-2 \pi d / h}}\|f\|_{\mathbf{H}^{1}\left(D_{d}\right)}+\frac{1}{1+N h} e^{-a h N}\right] .
$$

Proof. We recall that for any operator-valued function $f \in \mathbf{H}^{1}\left(D_{d}\right)$, the error $\eta_{N}(f, h)$ satisfies (3.17) and (3.18) due to Lemma 3.3. Assumption (4.4) now implies

$$
\begin{aligned}
\left\|\eta_{N}(f, h)\right\| & \leq\|\eta(f, h)\|+h \sum_{|k|>N}\|f(k h)\| \\
& \leq \frac{\exp (-\pi d / h)}{2 \sinh (\pi d / h)}\|f\|_{\mathbf{H}^{1}\left(D_{d}\right)}+c h \sum_{|k|>N} \frac{e^{-a|k h|}}{1+|k h|} .
\end{aligned}
$$

For the last sum we use the simple estimate

$$
\sum_{|k|>N} \frac{e^{-a|k h|}}{1+|k h|}=2 \sum_{k=N+1}^{\infty} \frac{e^{-a k h}}{1+k h} \leq \frac{2}{1+N h} \int_{N}^{\infty} e^{-a x h} d x=\frac{2 e^{-a h N}}{a h(1+N h)} .
$$

Therefore, it follows from (4.4) that $f \in \mathbf{H}^{1}\left(D_{d}\right)$ and

$$
\|f\|_{\mathbf{H}^{1}\left(D_{d}\right)} \leq 2 c \int_{-\infty}^{\infty} e^{-a|x|} d x \leq \frac{4 c}{a}
$$

which together with (4.6) and (4.7) implies the desired error estimate (4.5):

$$
\left\|\eta_{N}(f, h)\right\| \leq \frac{2 c}{a}\left[\frac{\exp (-\pi d / h)}{\sinh (\pi d / h)}+\frac{1}{1+N h} e^{-a h N}\right] .
$$

Now we can replace the integral (4.1) by the Sinc-quadrature rule $T_{N}$ (see (3.15) and get the approximation

$$
\mathcal{L}_{N}^{-\alpha}=-\frac{h}{2 \pi i} \sum_{k=-N}^{N} F_{2}(k h, \mathcal{L})
$$

The error analysis is given by the next theorem. Note that the class $\mathbf{E}_{S, R}$ is defined with respect to a parameter $d$ appearing in the strip $D_{d}$.

Theorem 4.3. Given an operator from the class $\mathbf{E}_{S, R}$, choose the integration curve $\Gamma_{I}=\left\{z=b \cosh \left(a_{1} \eta\right)+i \eta: \eta \in R\right\}$ with $b \in\left(0, \gamma_{0}\right), a_{1}>0$ and set $h=$ $\sqrt{\frac{2 \pi d}{a_{1}(\alpha-1) N}}$. Then

$$
\left\|\mathcal{L}^{-\alpha}-\mathcal{L}_{N}^{-\alpha}\right\| \leq \frac{2 c}{a(\alpha-1)}\left(e^{-2 \pi d / h}+\frac{1}{1+N h} e^{-a_{1}(\alpha-1) N h}\right) .
$$

Proof. In Lemma4.2 replace $f(x)$ by $F_{2}(x)$ and $a$ by $a_{1}(\alpha-1)$ (see Lemma4.1). 
The exponential convergence of our quadrature rule allows us to introduce the following algorithm for the approximation of negative powers $\mathcal{L}^{-\alpha}$ with a given tolerance $\varepsilon>0$.

Proposition 4.4. (a) Let $\varepsilon>0$ be given. In order to ensure $\left\|\mathcal{L}^{-\alpha}-\mathcal{L}_{N}^{-\alpha}\right\| \leq \varepsilon$, choose the parameters by

$$
\begin{aligned}
& a_{1}>a, \quad b \in\left(0, \gamma_{0}\right), \quad N=O(|\log \varepsilon|), \quad h=\sqrt{\frac{2 \pi d}{a_{1}(\alpha-1) N}}, \\
& z_{k}=b \cosh \left(a_{1} k h\right)+i k h \quad(k=-N, \ldots, N) .
\end{aligned}
$$

(b) $\mathcal{L}_{N}^{-\alpha}$ is a linear combination of $2 N+1$ resolvents like (3.25) so that parts (b) and (c) of Proposition 3.5 apply here too.

\section{Application to the Lyapunov solution operator}

We start with a matrix example. Let us consider the linear dynamical system of equations

$$
\frac{d X(t)}{d t}=A X(t)+X(t) B+G, \quad X(0)=X_{0}
$$

where $X(t), A, B, G \in \mathbb{R}^{n \times n}(A, B, G$ given constant matrices). The solution $X(t)$ is given by

$$
X(t)=e^{t A} X_{0} e^{t B}+\int_{0}^{t} e^{(t-s) A} G e^{(t-s) B} d s .
$$

If all eigenvalues of $A, B$ have negative real parts, then

$$
X(t) \rightarrow X_{\infty}=\int_{0}^{\infty} e^{t A} G e^{t B} d t \quad \text { as } t \rightarrow \infty,
$$

and the limit $X_{\infty}$ satisfies the matrix Lyapunov-Sylvester equation

$$
A X_{\infty}+X_{\infty} B+G=0 .
$$

We refer to 3 concerning the proof of (5.1) in the case of a matrix equation. The operator case considered below can be treated similarly.

5.1. Basic example. In the following, we generalise the corresponding results from 13. (applied to the matrix Lyapunov-Sylvester equation) to the case of the operatorvalued function $\mathcal{G}_{0}(z, \mathcal{L}, G)$. Specifically, we set $A=\mathcal{L}^{\top}, B=\mathcal{L}$ in (5.2), and we consider the solution of the operator Lyapunov equation: find a self-adjoint continuous operator $\mathcal{Z}: L^{2}(\Omega) \rightarrow V$ such that

$$
\mathcal{L}^{\top} \mathcal{Z}+\mathcal{Z} \mathcal{L}+G=0
$$

The solution $\mathcal{Z}$ of the operator Lyapunov equation is given by an integral representation on $\Gamma:=[0, \infty)$,

$$
\mathcal{Z}(\mathcal{L}):=\int_{0}^{\infty} \mathcal{G}_{0}(t, \mathcal{L}, G) d t
$$

where $\mathcal{G}_{0}(t, \mathcal{L}, G)$ is a continuous operator-valued function of $t \in[0, \infty)$, an elliptic second order operator $\mathcal{L}$ and a self-adjoint operator $G: L^{2}(\Omega) \rightarrow L^{2}(\Omega)$ defined by

$$
\mathcal{G}_{0}(z, \mathcal{L}, G):=e^{z \mathcal{L}^{\top}} G e^{z \mathcal{L}}
$$

(see [3] for the matrix case). 
Note that the continuous operators $\mathcal{L}, \mathcal{L}^{\top}: H_{0}^{1}(\Omega) \rightarrow H^{-1}(\Omega)$ admit continuous extensions by $\mathcal{L}: L^{2}(\Omega) \rightarrow V^{\prime}, \mathcal{L}^{\top}: V \rightarrow L^{2}(\Omega)$, where $V:=H^{2}(\Omega) \cap H_{0}^{1}(\Omega)$. As usual in control theory, we further assume $\Re$ e $\operatorname{sp}(-\mathcal{L}) \subset\left(\lambda_{0}, \infty\right)$.

For the analysis we need that $-\mathcal{L}$ is an elliptic operator of $\left(f_{S}, f_{R}\right)$-type with $f_{S}, f_{R}$ given by (2.3) such that

$$
s p(-\mathcal{L}) \subset S_{\mu}:=\left\{z \in \mathbb{C}:|\Im m z| \leq \mu, \Re e z>\lambda_{0}\right\}, \quad \lambda_{0}, \mu>0 .
$$

In particular, the latter condition implies that the elliptic operator $\mathcal{L}$ generates a strongly continuous semi-group $e^{t \mathcal{L}}$ such that

$$
\left\|e^{t \mathcal{L}}\right\| \leq C \frac{\mu^{2}}{\sqrt{t}} e^{-c \lambda_{0} t} \quad \text { for all } t \in[0, \infty) \quad(\|\cdot\|: \text { spectral operator norm }),
$$

with $C, c>0$ independent of $\lambda_{0}$ and $t$.

5.2. Cardinal series representations for analytic functions. Our goal is the construction and analysis of exponentially convergent quadrature rules for the integral (5.4). The construction consists of two steps: First, we approximate the integral by a sum of operators $\mathcal{G}_{0}\left(t_{k}, \mathcal{L}, G\right)$ computed at a few quadrature points $t_{k} \in[0, \infty)$, and then we approximate each operator exponential involved in $\mathcal{G}_{0}\left(t_{k}, \mathcal{L}, G\right)$ as in \$3.6. The resulting qudrature rule is similar to that in [13, Thm. 5] for the case of a matrix Lyapunov equation. Contrary to [13, here we consider the integral of an operator-valued function and therefore our proof is more technically involved; moreover, we apply the first-level quadrature to the scaled representation (see 5.7) and then use the new approximation scheme of 83.6

For the operator-valued function $\mathcal{Z}(\mathcal{L})$, we further use the scaled representation obtained by the substitution $t=u^{\alpha}$,

$$
\mathcal{Z}(\mathcal{L})=\int_{0}^{\infty} \mathcal{G}_{\alpha}(t, \mathcal{L}, G) d t:=\int_{0}^{\infty} g_{\alpha}\left(t, \mathcal{L}^{\top}\right) G g_{\alpha}(t, \mathcal{L}) d t
$$

where

$$
g_{\alpha}(z, \lambda):=\sqrt{1+2 \alpha} z^{\alpha} e^{z^{1+2 \alpha} \lambda}, \quad \alpha \geq 0,
$$

so that (5.4) corresponds to the case $\alpha=0$.

First, we recall some auxiliary approximation results for holomorphic functions based on the Sinc-approximation. Let the region ${ }^{3} \mathcal{D}_{d}$ (see Figure 3i) for a given $d \in(0, \pi / 2]$ be defined by

$$
\mathcal{D}_{d}:=\{z \in \mathbb{C}:|\arg (\sinh z)|<d\} .
$$

We denote by $H^{1}\left(\mathcal{D}_{d}\right)$ the family of functions that are analytic in $\mathcal{D}_{d}$ and that satisfy

$$
N_{1}\left(f, \mathcal{D}_{d}\right):=\int_{\partial \mathcal{D}_{d}}|f(z)||d z|<\infty .
$$

Now, for $\alpha, \beta \in(0,1]$, introduce the space

$$
L_{\alpha, \beta}\left(\mathcal{D}_{d}\right):=\left\{f \in H^{1}\left(\mathcal{D}_{d}\right):|f(z)| \leq C\left(\frac{|z|}{1+|z|}\right)^{\alpha} e^{-\beta \Re e z} \quad \text { for all } z \in \mathcal{D}_{d}\right\}
$$

(cf. [22]). We set

$$
\phi(z):=\log \{\sinh (z)\},
$$

\footnotetext{
${ }^{3}$ This is the domain called $\mathcal{D}_{d}^{3}$ in 22 . Note that it is different from the strip $D_{d}$ in (3.4).
} 


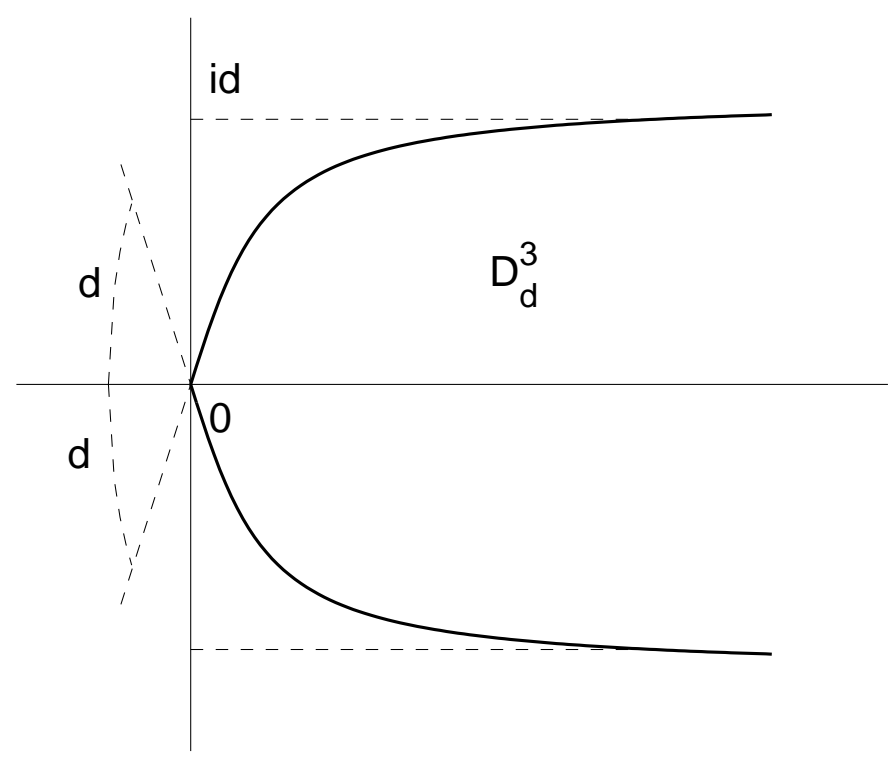

Figure 3 . The region $\mathcal{D}_{d}$

hence

$$
\phi^{\prime}(z)=\frac{1}{\tanh (z)}, \quad z=\phi^{-1}(w)=\log \left(e^{w}+\sqrt{1+e^{2 w}}\right),
$$

where $\phi(z)$ is the conformal map of $\mathcal{D}_{d}$ onto the infinite strip $D_{d}$ defined by (3.4).

For any $f \in H^{1}\left(\mathcal{D}_{d}\right)$, we introduce the integral representation for the error $\mathcal{I}$ arising in the cardinal series expansions

$$
\frac{f(z)}{\phi^{\prime}(z)}=\sum_{k=-\infty}^{\infty} \frac{f\left(t_{k}\right)}{\phi^{\prime}\left(t_{k}\right)} S(k, h)(\phi(z))+\mathcal{I}\left[\frac{f}{\phi^{\prime}}\right](z) \quad \text { for all } z \in \mathcal{D}_{d}
$$

(see 22] for more details) with

$$
\mathcal{I}[\psi](z):=\frac{\sin [\pi \phi(z) / h]}{2 \pi i} \int_{\partial \mathcal{D}_{d}} \frac{\psi(\zeta) \phi^{\prime}(\zeta) d \zeta}{[\phi(\zeta)-\phi(z)] \sin [\pi \phi(\zeta) / h]} .
$$

Similarly, if $\phi^{\prime} f \in H^{1}\left(\mathcal{D}_{d}\right)$, then

$$
f(z)=\sum_{k=-\infty}^{\infty} f\left(t_{k}\right) S(k, h)(\phi(z))+\mathcal{I}[f](z) \quad \text { for all } z \in \mathcal{D}_{d} .
$$

It is easy to check that

$$
g_{\alpha}(\cdot, \lambda) \in L_{\alpha, \beta}\left(\mathcal{D}_{d}\right), \quad \beta \geq \min \left\{1, b_{0}\right\} \quad \text { for } \lambda \in \Gamma_{\mathcal{L}},
$$

where, with given $b_{0}, a>0, \Gamma_{\mathcal{L}}:=\left\{z=\xi+i \eta: \xi=-b_{0}-a \eta^{2}, \eta \in(-\infty, \infty)\right\}$ is the integration parabola used in the proof of Lemma 5.2 below. Due to [22, Lemma 4.2.4] we know that (5.13) implies

$$
\phi^{\prime}(\cdot) g_{\alpha}(\cdot, \lambda) \in H^{1}\left(\mathcal{D}_{d}\right) .
$$

The next lemma proves a uniform bound on the value $N_{1}$ in (5.9) with respect to $\lambda \in \Gamma_{\mathcal{L}}$. 
Lemma 5.1. It holds that $g_{\alpha}(\cdot, \lambda) \in L_{\alpha, \beta}\left(\mathcal{D}_{d}\right)$. Moreover, $\phi^{\prime} g_{\alpha}(\cdot, \lambda) \in H^{1}\left(\mathcal{D}_{d}\right)$, where the corresponding value $N_{1}\left(\phi^{\prime} g_{\alpha}(\cdot, \lambda), \mathcal{D}_{d}\right)$ defined by (5.9) is uniformly bounded in $\lambda \in \Gamma_{\mathcal{L}}$.

Proof. The first assertion is easy to check. Our proof of the second statement is similar to that in [22, Lemma 4.2.4]. We have to prove that

$$
N_{1}\left(\phi^{\prime} g_{\alpha}(\cdot, \lambda), \mathcal{D}_{d}\right):=\int_{\partial \mathcal{D}_{d}}\left|\phi^{\prime}(z) g_{\alpha}(z, \lambda)\right||d z|<C<\infty,
$$

where $C$ does not depend on $\lambda$. If $z \in \partial \mathcal{D}_{d}$, then $\phi(z)=t+i \tau$ where $t=\Re e \phi(z)$, $\tau=\Im m \phi(z)= \pm d$. Thus $d z=\left\{1 / \phi^{\prime}(z)\right\} d t$ and $z=\phi^{-1}(t \pm i d)$ implying $|z|=$ $O(t), t \rightarrow \infty$, and

$$
\begin{aligned}
& \int_{\partial \mathcal{D}_{d}}\left|\phi^{\prime}(z) g_{\alpha}(z, \lambda)\right||d z| \\
& \quad \leq C \int_{\mathbb{R}} \frac{|t|^{\alpha}}{(1+|t|)^{\alpha}}\left|e^{\log ^{2}\left(e^{t \pm i d}+\sqrt{\left.1+e^{2(t \pm i d)}\right)}\left(-b_{0}-a \eta^{2}+i \eta\right)\right.}\right| d t \leq C\left(b_{0}, a\right)<\infty .
\end{aligned}
$$

Then (5.15) follows.

Due to Lemma 5.1, we can substitute $f(z)=g_{\alpha}(z, \lambda)$ in both (5.10) and (5.12).

5.3. First quadrature applied to $\mathcal{Z}(\mathcal{L})$. In the sequel, the value of $d$ involved in $\mathcal{D}_{d}$ (cf. (5.8)) will be chosen from the interval $0<d<\frac{\pi}{2(1+2 \alpha)}$, where we further fix the parameter $\alpha=1 / 2$. Without loss of generality we can assume $\beta=\min \left\{1, \lambda_{0} / 2\right\} \leq \alpha=1 / 2$ and then choose the parameter $b_{0}$ that defines the integrating parabola $\Gamma_{\mathcal{L}}$ by $b_{0}=\lambda_{0} / 2$. This choice also implies $a<\frac{\lambda_{0}}{2 \mu^{2}}$. Since $\alpha=1 / 2$ is fixed, we futher omit the subscript $\alpha$ in $g_{\alpha}$ and set $g=g_{1 / 2}$. Now we are in a position to derive the quadrature rule.

Lemma 5.2. Let the spectrum of $\mathcal{L}$ lie in the strip $S_{\mu}$ defined by (5.5). Given $N \in \mathbb{N}$ and $\beta=\min \left\{1, \lambda_{0} / 2\right\}$, with the proper choice of $h>0, t_{k} \in[0, \infty)$, and $M$ by

$$
h=\sqrt[3]{\frac{\pi d}{2 \lambda_{0} N^{2}}}, \quad t_{k}=\log \left(e^{k h}+\sqrt{1+e^{2 k h}}\right), \quad M=\lceil 2 \beta N\rceil,
$$

the integral $\mathcal{Z}(\mathcal{L})=\int_{0}^{\infty} \mathcal{G}_{0}(t, \mathcal{L}, G) d t$ allows an exponentially convergent quadrature rule

$$
\mathcal{Z}_{N}(\mathcal{L}):=h \sum_{k=-M}^{N} \tanh \left(t_{k}\right) \mathcal{G}_{\frac{1}{2}}\left(t_{k}, \mathcal{L}, G\right),
$$

providing the error estimate

$$
\left\|\mathcal{Z}(\mathcal{L})-\mathcal{Z}_{N}(\mathcal{L})\right\| \leq C \mu^{2} e^{-\left(2 \pi \lambda_{0} d N\right)^{2 / 3}}
$$

with the constant $C$ independent of $N$ and with $\mu$ being half the width of the strip $S_{\mu}$ in (5.5).

Proof. In our proof we extend the results from [22, Example 4.2.11] to the case of operator-valued functions. The proof consists of three steps. 
Step 1 (Cardinal series representations for $g(\cdot, \mathcal{L})$ ). First we note that under the above assumptions on $\mathcal{L}$ the operator-valued function $g(\cdot, \mathcal{L})$ can be represented by using the uniformly convergent Dunford-Cauchy integral

$$
g(z, \mathcal{L})=\frac{1}{2 \pi i} \int_{\Gamma_{\mathcal{L}}} g(z, \xi)(\xi I-\mathcal{L})^{-1} d \xi \quad \text { for } z \in \mathcal{D}_{d}
$$

where $\Gamma_{\mathcal{L}}$ is the parabola containing $\operatorname{sp}(\mathcal{L})$ as above. In fact, it is easy to check that for $\xi \in \Gamma_{\mathcal{L}}$,

$$
|g(z, \xi)| \leq C|z|^{1 / 2}\left|e^{z^{2}\left(-b_{0}-a \eta^{2}+i \eta\right)}\right|, \quad \eta \in(-\infty, \infty),
$$

where $\left|\arg z^{2}\right|<\pi / 2$, i.e., $\Re e z^{2}>0$, which ensures the convergence of the integral in (5.19). A similar representation holds for $\frac{g\left(z, \mathcal{L}^{\top}\right)}{\phi^{\prime}(z)}$. Therefore, due to $g(\cdot, \lambda) \in$ $H^{1}\left(\mathcal{D}_{d}\right), \lambda \in \Gamma_{\mathcal{L}}$ (see Lemma 5.1), the substitution of (5.10) into the DunfordCauchy integral leads to the identity

$$
\frac{g\left(t, \mathcal{L}^{\top}\right)}{\phi^{\prime}(t)}=\sum_{k=-\infty}^{\infty} \frac{g\left(t_{k}, \mathcal{L}^{\top}\right)}{\phi^{\prime}\left(t_{k}\right)} S(k, h)(\phi(t))+\mathcal{I}\left[\frac{g\left(\cdot, \mathcal{L}^{\top}\right)}{\phi^{\prime}}\right](t) \quad \text { for } t \in[0, \infty) .
$$

The convergence of the integral in the right-hand side of (5.20) follows from the estimate (for $\zeta \in \mathcal{D}_{d}, \zeta=t+i \tau,|\tau| \leq d$ )

$$
\left\|g\left(\zeta, \mathcal{L}^{\top}\right)\right\| \leq C \frac{e^{-b_{0} t^{2}}}{a \sqrt{t}} .
$$

To prove (5.21), first we consider the case $|\zeta| \rightarrow \infty$, i.e., $t \rightarrow \infty$, and we obtain

$$
\begin{aligned}
\left\|g\left(\zeta, \mathcal{L}^{\top}\right)\right\| & \leq C \int_{\Gamma_{\mathcal{L}}}|g(\zeta, \xi)| \|(\xi I-\mathcal{L})^{-1}|||d \xi| \\
& \leq C t \int_{-\infty}^{\infty} \frac{|-2 a \eta+i|}{1+\sqrt{a}|\eta|}\left|e^{\left(t^{2}-\tau^{2}+2 i t \tau\right)\left(-b_{0}-a \eta^{2}+i \eta\right)}\right| d \eta \\
& \leq C t e^{-b_{0}\left(t^{2}-d^{2}\right)} \int_{-\infty}^{\infty} e^{\left(d^{2}-t^{2}\right) \eta^{2}-2 t \tau \eta} d \eta .
\end{aligned}
$$

To study the case $\zeta \rightarrow 0$, we note that due to the bound on $d>0$, it holds that $|\tau| \leq \tan (d) t, t \rightarrow 0$ with $\tan (d)<1$. Then we obtain

$$
\begin{aligned}
\left\|g\left(\zeta, \mathcal{L}^{\top}\right)\right\| & \leq C \int_{\Gamma_{\mathcal{L}}}|g(\zeta, \xi)|\left\|(\xi I-\mathcal{L})^{-1}\right\||d \xi| \\
& \leq C \int_{-\infty}^{\infty} \sqrt{t} \frac{|-2 a \eta+i|}{1+\sqrt{a}|\eta|}\left|e^{c t^{2}\left(-b_{0}-a \eta^{2}+i \eta\right)}\right| d \eta \\
& \leq C \sqrt{t} e^{-b_{0} t^{2}} \int_{-\infty}^{\infty}\left|e^{c t^{2}\left(-a \eta^{2}+i \eta\right)}\right| d \eta \\
& \leq C \sqrt{t} e^{-b_{0} t^{2}} \int_{-\infty}^{\infty} e^{-a c t^{2} \eta^{2}} d \eta \\
& \leq C \sqrt{t} e^{-b_{0} t^{2}} \int_{0}^{\infty} \frac{1}{2 t^{2} \eta} e^{-a u} d u \\
& \leq C \frac{e^{-b_{0} t^{2}}}{\sqrt{t}} \int_{0}^{\infty} \frac{1}{\sqrt{u}} e^{-a u} d u \leq C \frac{e^{-b_{0} t^{2}}}{a \sqrt{t}}
\end{aligned}
$$


which shows that the integrand in (5.20) is $L^{1}$-integrable.

On the other hand, by the same arguments the property $\phi^{\prime} g(\cdot, \lambda) \in H^{1}\left(\mathcal{D}_{d}\right)$ (see Lemma 5.1) leads to the representation (see (5.12))

$$
g(t, \mathcal{L})=\sum_{k=-\infty}^{\infty} g\left(t_{k}, \mathcal{L}\right) S(k, h)(\phi(t))+\mathcal{I}[g(\cdot, \mathcal{L})](t) \quad \text { for } t \in[0, \infty)
$$

Step 2 (Quadrature with an explicit error representation). We multiply (5.20) by $\phi^{\prime}(z)$ and insert the result together with (5.24) into the integral to be approximated and we obtain

$$
\int_{0}^{\infty} \mathcal{G}_{1 / 2}(t, \mathcal{L}, G) d t:=\int_{0}^{\infty} g\left(t, \mathcal{L}^{\top}\right) G g(t, \mathcal{L}) d t=B(\mathcal{L}, G)+I_{1}(\mathcal{L}, G)+I_{2}(\mathcal{L}, G),
$$

where

$$
\begin{aligned}
B(\mathcal{L}, G) & :=\sum_{k=-\infty}^{\infty} \sum_{m=-\infty}^{\infty} \frac{g\left(t_{k}, \mathcal{L}^{\top}\right)}{\phi^{\prime}\left(t_{k}\right)} G g\left(t_{m}, \mathcal{L}\right) \int_{0}^{\infty} S(k, h)(\phi(t)) S(m, h)(\phi(t)) \phi^{\prime}(t) d t, \\
I_{1}(\mathcal{L}, G) & :=\int_{0}^{\infty} \phi^{\prime}(t) \mathcal{I}\left[\frac{g\left(\cdot, \mathcal{L}^{\top}\right)}{\phi^{\prime}}\right](t) G g(t, \mathcal{L}) d t \\
I_{2}(\mathcal{L}, G) & :=\int_{0}^{\infty}\left(g\left(t, \mathcal{L}^{\top}\right)-\phi^{\prime}(t) \mathcal{I}\left[\frac{g\left(\cdot, \mathcal{L}^{\top}\right)}{\phi^{\prime}}\right](t)\right) G \mathcal{I}[g(\cdot, \mathcal{L})](t) d t .
\end{aligned}
$$

Due to the orthogonality relation

$$
\int_{0}^{\infty} S(k, h)(\phi(z)) S(m, h)(\phi(z)) \phi^{\prime}(z) d z=h \delta_{k-m} \quad\left(\delta_{k-m}: \text { Kronecker symbol }\right)
$$

for Sinc-functions (see [22]), we can reduce the double sum in $B(\mathcal{L}, G)$ to

$$
B(\mathcal{L}, G)=h \sum_{k=-\infty}^{\infty} \frac{g\left(t_{k}, \mathcal{L}^{\top}\right)}{\phi^{\prime}\left(t_{k}\right)} G g\left(t_{k}, \mathcal{L}\right)
$$

Step 3 (Final error estimate). Using the relation $\phi^{\prime}\left(t_{k}\right)^{-1}=\tanh \left(t_{k}\right)$ which proves $\left|\phi^{\prime}\left(t_{k}\right)^{-1}\right|=O(1)$ as $t_{k} \rightarrow \infty$ and truncating the infinite sum $B(\mathcal{L}, G)$ from (5.26), we obtain the quadrature rule for the integral involving $\mathcal{G}_{1 / 2}$,

$$
\mathcal{G}_{N}:=h \sum_{k=-M}^{N} \tanh \left(t_{k}\right) g\left(t_{k}, \mathcal{L}^{\top}\right) G g\left(t_{k}, \mathcal{L}\right) .
$$

Due to 5.25 we readily obtain

$$
A_{0}:=\left\|\int_{0}^{\infty} \mathcal{G}_{1 / 2}(t, \mathcal{L}, G) d t-B(\mathcal{L}, G)\right\| \leq\left\|I_{1}(\mathcal{L}, G)\right\|+\left\|I_{2}(\mathcal{L}, G)\right\|,
$$

where the integrals $I_{1}(\mathcal{L}, G)$ and $I_{2}(\mathcal{L}, G)$ can be estimated by using (5.11). Taking into account that for $z \in \mathbb{R}$ and $\zeta \in \partial \mathcal{D}_{d}$ it holds that $\Im m \phi(\zeta)= \pm d$ and $-d<$ $\Im m \phi(z)<d$, we conclude that

$$
\begin{aligned}
|\sin (\pi \phi(\zeta) / h)| & \geq \sinh (\pi d / h), \\
|\sin (\pi \phi(z) / h)| & \leq \cosh [(\pi / h)\{\Im m \phi(z)\}], \\
|\phi(\zeta)-\phi(z)| & \geq d-|\Im m \phi(z)|
\end{aligned}
$$


(see [22]). Indeed, one can easily verify that

$$
\begin{aligned}
& \left\|I_{1}(\mathcal{L}, G)\right\| \leq C\|G\| \int_{0}^{\infty} \sqrt{t} e^{-a_{0} t^{2}}\left|\phi^{\prime}(t)\right|\left\|\mathcal{I}\left[\frac{g\left(\cdot, \mathcal{L}^{\top}\right)}{\phi^{\prime}}\right](t)\right\| d t \\
& \quad \leq C\|G\| \int_{\partial \mathcal{D}_{d}}\left\|g\left(\zeta, \mathcal{L}^{\top}\right)\right\| d \zeta \int_{0}^{\infty} \sqrt{t} e^{-a_{0} t^{2}} \frac{\sin [\pi \phi(t) / h]}{\sinh (\pi d / h)} \max _{\zeta \in \mathcal{D}_{d}} \frac{1}{|\phi(\zeta)-\phi(t)|} d t \\
& \quad \leq C N_{1}\left(g\left(\cdot, \mathcal{L}^{\top}\right), \mathcal{D}_{d}\right)\|G\| e^{-\pi d / h} .
\end{aligned}
$$

The bound for $I_{2}(\mathcal{L}, G)$ is more involved. We split $I_{2}$ into two terms. Similarly to above we derive

$$
\left\|\int_{0}^{\infty} g\left(t, \mathcal{L}^{\top}\right) G \mathcal{I}[g(\cdot, \mathcal{L})](t) d t\right\| \leq C N_{1}\left(\phi^{\prime} g(\cdot, \mathcal{L}), \mathcal{D}_{d}\right)\|G\| e^{-\pi d / h}
$$

by taking into account that $\left|\phi^{\prime}(t)\right| \rightarrow 1$ as $t \rightarrow \infty$. Using the inequality (5.29), the asymptotic behaviour $\Re e \phi(t) \sim t$ as $t \rightarrow \infty$ and $\Im m \phi(t)=0$ for $t \in[0, \infty)$, we get

$$
\begin{aligned}
\| \int_{0}^{\infty} \mathcal{I} & {[g(\cdot, \mathcal{L})](t) G \mathcal{I}\left[\frac{g\left(\cdot, \mathcal{L}^{\top}\right)}{\phi^{\prime}}\right](t) \phi^{\prime}(t) d t \| } \\
\leq & C e^{-2 \pi d / h} N_{1}\left(g\left(\cdot, \mathcal{L}^{\top}\right), \mathcal{D}_{d}\right) N_{1}\left(\phi^{\prime} g(\cdot, \mathcal{L}), \mathcal{D}_{d}\right)\|G\| \\
& \times \sup _{\zeta \in \partial \mathcal{D}_{d}} \int_{0}^{\infty} \frac{d t}{d^{2}+|\Re e \phi(\zeta)-\Re e \phi(t)|^{2}}
\end{aligned}
$$

for the second term, where the corresponding integral over $[0, \infty)$ is uniformly bounded with respect $\zeta \in \partial \mathcal{D}_{d}$.

Finally, we estimate the sum representing the error $\delta_{N}$ of the quadrature rule,

$$
\delta_{N}:=\left\|\int_{0}^{\infty} \mathcal{G}_{0}(t, \mathcal{L}, G) d t-h \sum_{k=-M}^{N} \frac{\mathcal{G}_{1 / 2}\left(t_{k}, \mathcal{L}, G\right)}{\phi^{\prime}\left(t_{k}\right)}\right\| \leq A_{0}+A_{1}+A_{2},
$$

where

$$
\begin{aligned}
& \left.A_{0} \leq C e^{-\pi d / h} N_{1}\left(g\left(\cdot, \mathcal{L}^{\top}\right), \mathcal{D}_{d}\right) N_{1}\left(\phi^{\prime} g(\cdot, \mathcal{L})\right)\|G\| \quad \text { for } A_{0} \text { from (15.28) }\right) \\
& A_{1}:=\left\|h \sum_{k=-\infty}^{-M-1} \frac{\mathcal{G}_{1 / 2}\left(t_{k}, \mathcal{L}, G\right)}{\phi^{\prime}\left(t_{k}\right)}\right\|, \quad A_{2}:=\left\|h \sum_{k=N+1}^{\infty} \frac{\mathcal{G}_{1 / 2}\left(t_{k}, \mathcal{L}, G\right)}{\phi^{\prime}\left(t_{k}\right)}\right\| .
\end{aligned}
$$

Next, we consider $A_{2}$ (the treatment of $A_{1}$ is similar). Due to the estimate

$$
\begin{aligned}
\left\|\frac{\mathcal{G}_{1 / 2}\left(t_{k}, \mathcal{L}, G\right)}{\phi^{\prime}\left(t_{k}\right)}\right\| & \leq C\|G\|\left\|\frac{g\left(t_{k}, \mathcal{L}^{\top}\right)}{\phi^{\prime}\left(t_{k}\right)}\right\|\left\|g\left(t_{k}, \mathcal{L}\right)\right\| \\
& \leq C\|G\| t_{k} e^{-2 \lambda_{0} t_{k}^{2}} \leq C\|G\| e^{-2 \lambda_{0}(k h)^{2}},
\end{aligned}
$$

(see [22, (4.1.7)]), we obtain

$$
A_{2} \leq C h \sum_{k=N+1}^{\infty} e^{-2 \lambda_{0}(k h)^{2}} \leq C h \int_{N}^{\infty} e^{-2 \lambda_{0}(x h)^{2}} d x=\frac{C h}{2 \lambda_{0} h^{2} N} e^{-2 \lambda_{0} h^{2} N^{2}},
$$

where the constant $C=C(\mathcal{L}, G)$ can be estimated by $C \leq c\|G\|$. Now we derive $\delta_{N} \leq C e^{-\pi d / h} N_{1}\left(g\left(\cdot, \mathcal{L}^{\top}\right), \mathcal{D}_{d}\right) N_{1}\left(\phi^{\prime} g(\cdot, \mathcal{L})\right)\|G\|+C\|G\|\left(e^{-2 \lambda_{0} h^{2} M^{2}}+e^{-2 \lambda_{0} h^{2} N^{2}}\right)$, 
that proves the result for $\mathcal{G}_{0}$, taking into account that according to (5.22) and (5.23)

$$
N_{1}\left(g\left(\cdot, \mathcal{L}^{\top}\right), \mathcal{D}_{d}\right) \leq C a^{-1}, \quad N_{1}\left(\phi^{\prime} g(\cdot, \mathcal{L}), \mathcal{D}_{d}\right) \leq C a^{-1}
$$

The choice of the parameters $h$ and $M$ by (15.16) leads to the desired error estimate.

Remark 5.3. Note that setting $\mathcal{L}=-\beta I, G=I$ leads to the exponentially convergent qudrature rule for the integral of a holomorphic function $g(z):=\mathcal{G}_{0}(z,-\beta I, I)$ over $[0, \infty)$, which is a particular case of [22, Theorem 4.2.11].

Lemma 5.2 already provides a low rank approximation to the solution of the Lyapunov equation in the case where a right-hand side $G$ has this property. In fact, let us assume that $G$ has a separable representation consisting of $k_{G}$ terms, i.e.,

$$
G:=\sum_{\alpha=1}^{k_{G}} a_{\alpha} * f_{\alpha}
$$

where $f_{\alpha}: L^{2}(\Omega) \rightarrow \mathbb{R}$ are linear continuous functionals, while $a_{\alpha} \in L^{2}(\Omega)$ are functions on $\Omega$. Substitution of the above representation into $\mathcal{G}_{0}\left(t_{k}, \mathcal{L}, G\right)=e^{t_{k} \mathcal{L}^{\top}} G e^{t_{k} \mathcal{L}}$ also shows that $\mathcal{G}_{0}\left(t_{k}, \mathcal{L}, G\right)$ is separable with $k_{G}$ terms. Due to (5.17), $\mathcal{Z}_{N}(\mathcal{L})$ is separable with $k=k_{G}(N+M+1)$ terms (in the matrix case, this is equivalent to $\left.\operatorname{rank} \mathcal{Z}_{N}(\mathcal{L}) \leq k\right)$

5.4. Second quadrature applied to $\mathcal{Z}_{N}(\mathcal{L})$. We proceed with the approximation of the individual terms $\mathcal{G}_{1 / 2}\left(t_{k}, \mathcal{L}, G\right)$ in (5.17). For this purpose, we apply the basic construction from $\$ 3.4$ modified by a proper translation transform explained below. We use the symbol $A$ for both $\mathcal{L}$ and $\mathcal{L}^{\top}$.

We recall that with a given elliptic operator $A$ and for the described choice of the parameters $z_{p}, h, c_{p}$, the quadrature

$$
\exp _{L}(A)=\sum_{p=-L}^{L} c_{p} e^{-z_{p}}\left(z_{p} I-A\right)^{-1} \quad(\text { see }(3.25))
$$

provides exponential convergence (cf. (3.24)). To adapt the above approximation to our particular situation, we include the parameter $t_{k}$ into the operator by setting $A_{k}:=t_{k}^{2} \mathcal{L}$, which then leads to the bound $\lambda_{\min }\left(A_{k}\right)=O\left(t_{k}^{2}\right)$. Due to the factor $\frac{1}{t}$ in (3.24), the error estimate deteriorates when $t_{k} \rightarrow 0$. To obtain uniform convergence with respect to $t_{k} \rightarrow 0$, we use a simple shift of the spectrum,

$$
e^{A_{k}}=e^{-1} \cdot e^{B_{k}} \quad \text { for } B_{k}:=A_{k}+I,
$$

ensuring that $\lambda_{\min }\left(B_{k}\right)=O(1)>0$. Now we apply the quadrature (5.31) to the operator $B_{k}$, which leads to the uniform error estimate

$$
\left\|\exp \left(B_{k}\right)-\exp _{L}\left(B_{k}\right)\right\| \leq C e^{-L / \log L} \quad \text { for all } k=-M, \ldots, N,
$$

where the constant $C$ does not depend on $L$ and $k$. With this procedure, we arrive at the following product quadrature.

Theorem 5.4. Under the conditions of Lemma 5.2, the expression

$$
\mathcal{Z}_{N, L}(\mathcal{L}):=2 h \sum_{k=-M}^{N} t_{k} \tanh \left(t_{k}\right) \mathcal{S}_{L, k}^{\top} G \mathcal{S}_{L, k} \text { with } \mathcal{S}_{L, k}:=\sum_{p=-L}^{L} c_{p} e^{-1-z_{p}}\left(z_{p} I-B_{k}\right)^{-1}
$$


converges exponentially to $\mathcal{Z}(\mathcal{L})$,

$$
\left\|\mathcal{Z}(\mathcal{L})-\mathcal{Z}_{N, L}(\mathcal{L})\right\| \leq C\left[\mu^{2} e^{-\left(2 \pi \lambda_{0} d N\right)^{2 / 3}}+e^{-L / \log L}\right] \quad \text { as } N, L \rightarrow \infty .
$$

Proof. The combination of the result of Lemma 5.2 with the modified quadrature (5.31) leads to the desired bound.

\section{Approximation to the Weighted Cosine operator}

6.1. Integral representation of the weighted cosine operator. In this section we consider the integral representation of a so-called weighted cosine family generated by an $\left(f_{S}, f_{R}\right)$-type operator of class $\mathbf{E}_{R, S}$. We define the following classes of operator-valued functions:

$$
\begin{aligned}
& \mathbf{H}_{e^{-\alpha \eta}}^{0}\left(\mathbb{R}^{+}\right):=\mathbf{L}_{e^{-\alpha \eta}}^{2}\left(\mathbb{R}^{+}\right):=\left\{f: \int_{0}^{\infty}\|f(\eta)\|^{2} e^{-\alpha \eta} d \eta<\infty\right\}, \\
& \mathbf{H}_{e^{-\alpha \eta}}^{m}\left(\mathbb{R}^{+}\right):=\left\{f \in \mathbf{L}_{e^{-\alpha \eta}}^{2}\left(\mathbb{R}^{+}\right): \frac{d^{r} f}{d \eta^{r}} \in \mathbf{L}_{e^{-\alpha \eta}}^{2}\left(\mathbb{R}^{+}\right), 0 \leq r \leq m\right\} .
\end{aligned}
$$

The spaces $\mathbf{H}_{e^{-\alpha \eta}}^{m}\left(\mathbb{R}^{+}\right)$can also be defined as Hilbert spaces with the scalar product

$$
(f, g)_{m, e^{-\alpha \eta}}=\sum_{r=0}^{m} \int_{0}^{\infty}\left(\frac{d^{r} f(\eta)}{d \eta^{r}}, \frac{d^{r} g(\eta)}{d \eta^{r}}\right) e^{-\alpha \eta} d \eta
$$

and the corresponding norm $\|\cdot\|_{m, e^{-\alpha \eta}}$. The spaces $\mathbf{H}_{e^{-\alpha \eta}}^{m}\left(\mathbb{R}^{+}\right)$for noninteger $m$ are defined via interpolation (cf. [19]).

Let $\mathcal{L}$ be a linear, densely defined, closed operator of $\left(f_{S}, f_{R}\right)$-type in a Banach space $X$. The operator-valued function $C_{k} \equiv C_{k}(\cdot ; \mathcal{L}):=C(\cdot ; \mathcal{L}) \mathcal{L}^{-k}, k \in \mathbb{N}$, defines the family $\left\{C_{k}(t): t \in \mathbb{R}\right\}$ of weighted cosine operators. It satisfies the differential equation

$$
C_{k}^{\prime \prime}(t)+\mathcal{L} C_{k}(t)=0 \quad \text { for } t>0, \quad C_{k}(0)=\mathcal{L}^{-k}, \quad C_{k}^{\prime}(0)=\Theta,
$$

where $\Theta$ is the zero operator. The vector-valued solution $u(\cdot):(0, \infty) \rightarrow X$ of the hyperbolic differential equation

$$
u^{\prime \prime}+\mathcal{L} u=0, \quad u(0)=u_{0}, \quad u^{\prime}(0)=0
$$

with an initial value $u_{0} \in X$ can be represented as

$$
u(t)=C_{k}(t) \mathcal{L}^{k} u_{0} \text { for } t>0,
$$

provided that $u_{0} \in X$ belongs to the domain of the operator $\mathcal{L}^{k}$.

Lemma 6.1. Let $\mathcal{L}$ be an operator from the class $\mathbf{E}_{S, R}$ and $k>1$. Choose a curve (the integration curve) $\Gamma_{I}=\left\{z=\xi+i \eta: \xi=b \cosh \left(a_{1} \eta\right)\right\}$ with $a_{1}<a, b \in\left(0, \gamma_{0}\right)$. Then the operator $C_{k}(t, \mathcal{L})$ can be represented by the Dunford-Cauchy integral

$$
\begin{aligned}
C_{k}(t ; \mathcal{L}) & =\frac{1}{2 \pi i} \int_{\Gamma_{I}} z^{-k} \cos (\sqrt{z} t)(z I-\mathcal{L})^{-1} d z \\
& =-\frac{1}{\pi i} \int_{-\infty}^{\infty} e^{-a_{1}|\eta|} F(\eta, t) d \eta=-\frac{1}{a_{1} \pi i} \int_{0}^{\infty} e^{-\zeta} F_{c}(\zeta, t) d \zeta
\end{aligned}
$$


where, for $\zeta \in(0, \infty), \eta \in(-\infty, \infty)$,

$$
\begin{aligned}
& F(\eta, t)=e^{a_{1}|\eta|} z^{-k} \cos (\sqrt{z} t)\left(b a_{1} \sinh \left(a_{1} \eta\right)+i\right)(z I-\mathcal{L})^{-1} \\
& F_{c}(\zeta, t)=F\left(-\zeta / a_{1}, t\right)+F\left(\zeta / a_{1}, t\right) .
\end{aligned}
$$

The integrand $F_{c}(\cdot, t)$ belongs to the class $\mathbf{H}_{e^{-2 \eta}}^{k}(0, \infty)$.

Proof. The integration curve is given by $f_{I}(\eta)=b \cosh \left(a_{1} \eta\right)$. Using the formulae

$$
\begin{aligned}
& \sqrt{z}=\sqrt{\xi+i \eta}= \pm\left\{\sqrt{\frac{1}{2}\left[\xi+\sqrt{\xi^{2}+\eta^{2}}\right]}+i \operatorname{sign}(\eta) \sqrt{\frac{1}{2}\left[-\xi+\sqrt{\xi^{2}+\eta^{2}}\right]}\right\} \\
& = \pm\left\{\sqrt{\frac{1}{2}\left[\xi+\sqrt{\xi^{2}+\eta^{2}}\right]}+i \operatorname{sign}(\eta) \frac{|\eta|}{\sqrt{2} \sqrt{\xi+\sqrt{\xi^{2}+\eta^{2}}}}\right\} \text { with } \xi:=f_{I}(\eta) \\
& \cos (x \pm i y)=\cos (x) \cosh (y) \mp i \sin (x) \sinh (y) \\
& \sin (x \pm i y)=\sin (x) \cosh (y) \pm i \cos (x) \sinh (y) \\
& \cosh (x \pm i y)=\cos (y) \cosh (x) \pm i \sin (y) \sinh (x), \\
& \sinh (x \pm i y)=\cos (y) \sinh (x) \pm i \sin (y) \cosh (x), \\
& \text { we have for } z=b \cosh \left(a_{1} \eta\right)+i \eta \in \Gamma_{I}, \eta \in(-\infty, \infty), \text { that } \\
& \qquad \sqrt{z}= \pm\left(u_{s q}(\eta)+i \operatorname{sign}(\eta) v_{s q}(\eta)\right)
\end{aligned}
$$

with

$$
\begin{aligned}
u_{s q}(\eta) & =\sqrt{\frac{1}{2}\left[b \cosh \left(a_{1} \eta\right)+\sqrt{\left(b \cosh \left(a_{1} \eta\right)\right)^{2}+\eta^{2}}\right]}=\mathcal{O}\left(\sqrt{b \cosh \left(a_{1} \eta\right)}\right) \\
v_{s q}(\eta) & =\sqrt{\frac{1}{2}\left[-b \cosh \left(a_{1} \eta\right)+\sqrt{\left(b \cosh \left(a_{1} \eta\right)\right)^{2}+\eta^{2}}\right]} \\
& =\frac{|\eta|}{\sqrt{2} \sqrt{b \cosh \left(a_{1} \eta\right)+\sqrt{\left(b \cosh \left(a_{1} \eta\right)\right)^{2}+\eta^{2}}}}=\mathcal{O}\left(\frac{|\eta|}{2 \sqrt{b \cosh \left(a_{1} \eta\right)}}\right)
\end{aligned}
$$

Furthermore, we have

$$
\cos (\sqrt{z} t)=u_{c}(\eta, t)+i v_{c}(\eta, t)
$$

with

$$
\begin{aligned}
& u_{c}(\eta, t)=\cos \left(t u_{s q}(\eta)\right) \cosh \left(t \operatorname{sign}(\eta) v_{s q}(\eta)\right), \quad u_{c}(-\eta, t)=u_{c}(\eta, t), \\
& v_{c}(\eta, t)=\sin \left(t u_{s q}(\eta)\right) \sinh \left(t \operatorname{sign}(\eta) v_{s q}(\eta)\right), \quad v_{c}(-\eta, t)=-v_{c}(\eta, t) .
\end{aligned}
$$

It is easy to see that for $\eta \rightarrow \infty$,

$$
u_{s q}(\eta)=\mathcal{O}\left(\sqrt{b \cosh \left(a_{1} \eta\right)}\right), \quad v_{s q}(\eta)=\mathcal{O}\left(\eta / \sqrt{b \cosh \left(a_{1} \eta\right)}\right)
$$

and for a fixed $t$ and $\eta \rightarrow \infty$,

$$
u_{c}(\eta, t)=\mathcal{O}(1), \quad v_{c}(\eta, t)= \begin{cases}0 & t=0 \\ \mathcal{O}\left(\eta / \sqrt{b \cosh \left(a_{1} \eta\right)}\right) & t>0\end{cases}
$$

so that on the integration path it holds that

$$
\cos (\sqrt{z} t)=\mathcal{O}(1)+i \mathcal{O}\left(\eta / \sqrt{b \cosh \left(a_{1} \eta\right)}\right) .
$$


After parametrisation of the path $\Gamma_{I}$ we get

$$
\begin{aligned}
C_{k}(t) & =\frac{1}{2 \pi i} \int_{\Gamma_{I}} z^{-k} \cos (\sqrt{z} t)(z I-\mathcal{L})^{-1} d z \\
= & \frac{1}{2 \pi i} \int_{\infty}^{0} z^{-k}\left(u_{c}(\eta, t)-i v_{c}(\eta, t)\right)\left(b a_{1} \sinh \left(a_{1} \eta\right)+i\right)\left(b \cosh \left(a_{1} \eta\right)+i \eta-\mathcal{L}\right)^{-1} d \eta \\
& +\frac{1}{2 \pi i} \int_{0}^{-\infty} z^{-k}\left(u_{c}(\eta, t)-i v_{c}(\eta, t)\right)\left(b a_{1} \sinh \left(a_{1} \eta\right)+i\right) \\
& \times\left(b \cosh \left(a_{1} \eta\right)+i \eta-\mathcal{L}\right)^{-1} d \eta \\
= & -\frac{1}{\pi i} \int_{-\infty}^{\infty} e^{-a_{1}|\eta|} F(\eta, t) d \eta=-\frac{1}{a_{1} \pi i} \int_{0}^{\infty} e^{-\zeta} F_{c}(\zeta, t) d \zeta
\end{aligned}
$$

Since the operator $\mathcal{L}$ belongs to the class $\mathbf{E}_{S, R}$ and $\left\|(z I-\mathcal{L})^{-1}\right\| \leq 1 /|\Im m z|$, we get

$$
\left\|C_{k}(t)\right\| \leq C \int_{0}^{\infty}(1+\zeta)^{-1} e^{-(k-1) \zeta} d \zeta .
$$

The last integral converges for all $t \geq 0$ due to the assumption $k>1$.

Having in mind that

$$
\begin{gathered}
F_{c}(\zeta, t)=F(-\eta, t)+F(\eta, t), \\
F(\eta, t)=e^{a_{1}|\eta|} z^{-k} \cos (\sqrt{z} t)\left(b a_{1} \sinh \left(a_{1} \eta\right)+i\right)(z I-\mathcal{L})^{-1}
\end{gathered}
$$

with $z=b \cosh \left(a_{1} \eta\right)+i \eta, \eta \in(-\infty, \infty)$, we obtain after the first differentiation with respect to $\eta$ that

$$
\begin{aligned}
\frac{d}{d \eta} z^{-k} & =z^{-(k+1)}\left(b a_{1} \sinh \left(a_{1} \eta\right)+i\right), \\
\frac{d}{d \eta} \cos (\sqrt{z} t) & =\sin (\sqrt{z} t) \frac{t}{2 \sqrt{z}}\left(b a_{1} \sinh \left(a_{1} \eta\right)+i\right), \\
\frac{d}{d \eta}\left(b a_{1} \sinh \left(a_{1} \eta\right)+i\right) & =b a_{1}^{2} \cosh \left(a_{1} \eta\right), \\
\frac{d}{d \eta}(z I-\mathcal{L})^{-1} & =-(z I-\mathcal{L})^{-2}\left(b a_{1} \sinh \left(a_{1} \eta\right)+i\right) .
\end{aligned}
$$

Due to (6.3), the terms $\cos (\sqrt{z} t)$ and $\sin (\sqrt{z} t)$ remain bounded for $\eta \in(-\infty, \infty)$. Since $\left|\frac{d}{d \eta}(\sqrt{z} t) \cdot \frac{d}{d \eta} z\right| \approx e^{a_{1} \eta / 2}$, each differentiation of these terms increases the asymptotic behaviour by the factor $e^{a_{1} \eta / 2}$. Due to $|z| \approx e^{a_{1} \eta}$, the asymptotic behaviour of the terms $\left|z^{-m}\right|(m>0)$ is not changed by differentiation. The same holds for $\cos \left(a_{1} \eta\right)$ and $\sinh \left(a_{1} \eta\right)$. Since $\left\|(z I-\mathcal{L})^{-1}\right\| \leq c /(1+\eta)$, the asymptotic behaviour of the derivatives of $\left\|(z I-\mathcal{L})^{-m}\right\|$ becomes worse by the factor $e^{a_{1} \eta} /(1+\eta)$ with every further differentiation. Altogether, each differentiation leads to a worsening of the asymptotic behaviour of $F_{c}(\zeta, t)$ by $e^{\zeta} / \zeta$. Thus, since $\left\|F_{c}(\zeta, t)\right\| \leq c e^{-(k-1) \zeta} / \zeta$, we get that $F_{c} \in \mathbf{H}_{e^{-2 \eta}}^{k}$.

6.2. Gauss-Lobatto quadrature of Laguerre type. In this section we describe a Gauss-Lobatto quadrature for certain weighted improper integrals of operatorvalued functions. The proof of these results is completely analogous to that of [19] if we replace the modulus of functions by the corresponding operator norms. Let $\zeta_{0}=0$ and let the zeros $\zeta_{k}, k=1,2, \ldots, N$, of the Laguerre polynomial $\frac{d}{d x} L_{N+1}$ form the grid $\Omega_{N+1}$. Let $I_{N} u$ be the interpolation polynomial of $u(\zeta)$ with respect to 
the nodes $\Omega_{N+1}$. Using the interpolation polynomial, one can get the interpolatory quadrature formula

$$
I(u) \equiv \int_{0}^{\infty} e^{-\zeta} u(\zeta) d \zeta \approx Q_{N}(u) \equiv \sum_{p=0}^{N} \omega_{p} u\left(\zeta_{p}\right)
$$

which is exact for polynomials of degree $\leq N$. The quadrature coefficients are

$$
\omega_{p}=\frac{1}{(N+1) L_{N+1}^{2}\left(\zeta_{p}\right)} .
$$

The quadrature coefficients possess the stability property

$$
\sum_{p=0}^{N} \omega_{p} e^{(1-\epsilon) \zeta_{p}} \leq \frac{1}{\epsilon} \quad \text { for all } \epsilon \in(0,1] .
$$

The following estimates

$$
\begin{aligned}
\left\|u-I_{N} u\right\|_{\mu, e^{-2(1+\epsilon) \eta}} & \leq C_{\epsilon} N^{\mu-(\sigma / 2)+(1 / 2)}\|u\|_{\sigma, e^{-2 \eta}}, \\
\left\|I(u)-Q_{N}(u)\right\|_{\mu, e^{-2(1+\epsilon) \eta}} & \leq C_{\epsilon} N^{\mu-(\sigma / 2)+(1 / 2)}\|u\|_{\sigma, e^{-2 \eta}}
\end{aligned}
$$

hold for all $\sigma>1 / 2,0 \leq \mu \leq \sigma, \epsilon>0$ (cf. [19]).

Note that the orthogonal projection operator $\pi: H_{e^{-\beta \eta}}^{\sigma} \rightarrow P_{N}$ with respect to the corresponding scalar product satisfies

$$
\|u-\pi u\|_{\mu, e^{-(\beta+\epsilon) \eta}} \leq C_{\beta, \epsilon} N^{\mu-(\sigma / 2)}\|u\|_{\sigma, e^{-\beta \eta}}
$$

(cf. [19]), i.e., the estimates (6.5) are asymptotically optimal up to a factor $N^{1 / 2}$. The representation given by Lemma 6.1 allows us to introduce the following algorithm for the approximation of the weighted cosine function.

Proposition 6.2. Let $t$ and $N$ be given. Define $z_{p}(p=0, \ldots, N)$ by $z_{p}=$ $b \cosh \left(\zeta_{p}\right)-i \zeta_{p} / a_{1}$, where $\zeta_{0}=0$, and $\zeta_{p}, p=1, \ldots, N$, are the zeros of the first derivative of the Laguerre polynomial $L_{N+1}(\zeta)$. Let $\omega_{p}$ be defined by (6.4). Then the approximation $C_{k, N}(t ; \mathcal{L})$ is the sum

$$
C_{k, N}(t ; \mathcal{L})=\sum_{p=0}^{N} \gamma_{p}(t)\left(z_{p} I-\mathcal{L}\right)^{-1}
$$

with $\gamma_{p}(t):=\omega_{p} z_{p}^{-k} \cos \left(\sqrt{z_{p}} t\right)\left[e^{\zeta_{p}}\left(b a_{1} \sinh \left(\zeta_{p}\right)+i\right)+e^{-\zeta_{p}}\left(-b a_{1} \sinh \left(\zeta_{p}\right)+i\right)\right]$.

Due to estimates (6.5) we have the error bound

$$
\left\|C_{k}(t ; \mathcal{L})-C_{k, N}(t ; \mathcal{L})\right\|_{\mu, e^{-2(1+\epsilon) \eta}} \leq C N^{\mu-(k-1) / 2}\|u\|_{k, e^{-2 \eta}}
$$

for $k>1,0 \leq \mu \leq(k-1) / 2, \epsilon>0$.

\section{ApPENDiX}

7.1. Examples of $\left(f_{S}, f_{R}\right)$-type operators with exponentially increasing $f_{S}$. As a first example let us consider a self-adjoint, positive definite operator $A$ in a Hilbert space $H$ with $(A u, u)>\gamma_{0}(u, u)$. Any such operator is of $\left(f_{S}, f_{R}\right)$-type with

$$
f_{S}(\eta)=\gamma_{0} \cosh (a \eta), \quad f_{R}(z)= \begin{cases}1 /|\Im m z| & \text { if } \Re e z \gamma_{0}>0, \\ \left|z-z_{0}\right| & \text { otherwise }\end{cases}
$$


where $a$ is an arbitrary positive number and $z_{0}=\left(\gamma_{0}, 0\right)$. Here the function $f_{S}$ possesses exponential growth.

As a second example of an operator with nonreal spectrum one can consider the operator arising in the problem on small motions of a viscoelastic fluid in an arbitrary domain $\Omega \in \mathbb{R}^{3}$ (cf. [1]).

This problem is to find the velocity field $\vec{u}(t, x)$ and the pressure field $p(t, x)$ satisfying the following system of equations with boundary and initial conditions

$$
\begin{aligned}
& \frac{\partial \vec{u}}{\partial t}=-\rho^{-1} \nabla p+\nu I_{0}(t)(\Delta \vec{u})+\vec{f}, \quad \operatorname{div} \vec{u}=0 \quad \text { in } \Omega, \\
& \left(I_{0}(t) \vec{v}\right)(t, x):=\vec{v}(t, x)+\sum_{j=1}^{m} \alpha_{j} \int_{0}^{t} e^{-\gamma_{j}(t-s)} \vec{v}(s, x) d s, \\
& \vec{u}=\overrightarrow{0} \text { on } S=\partial \Omega, \quad \vec{u}(0, x)=\vec{u}^{0}(x),
\end{aligned}
$$

where $\nu>0$ is the kinematic viscosity coefficient, $\rho>0$ is the density of the fluid, $\alpha_{j}>0, j=1, \ldots, m, 0<\gamma_{1}<\cdots<\gamma_{m}$, and $\vec{f}=\vec{f}(t, x)$ is the density of the small field of mass forces added to the gravitational field.

We assume that for every $t, \vec{u}(t, x)$ and $\nabla p(t, x)$ are elements of the Hilbert space $\vec{L}_{2}(\Omega)$ of vector-valued functions, equipped with the inner product

$$
(\vec{u}, \vec{v})_{\Omega}:=\int_{\Omega} \sum_{k=1}^{3} u_{k}(x) \overline{v_{k}(x)} d \Omega .
$$

We use the orthogonal decomposition

$$
\begin{gathered}
\vec{L}_{2}(\Omega)=\vec{J}_{0}(\Omega) \oplus \vec{G}(\Omega), \quad G(\vec{\Omega}):=\left\{\vec{v} \in \vec{L}_{2}(\Omega): \vec{v}=\nabla p\right\}, \\
\vec{J}_{0}(\Omega):=\left\{\vec{u} \in \vec{L}_{2}(\Omega): \operatorname{div} \vec{u}=0 \text { in } \Omega, u_{n}:=\vec{u} \cdot \vec{n}=0 \text { on } \partial \Omega\right\},
\end{gathered}
$$

where $\vec{n}$ is the unit normal vector to $\partial \Omega$ and $\vec{u}$ and $u_{n}$ are regarded as distributions of finite order. By (7.2), we have $\vec{u}(t, x) \in \vec{J}_{0}(\Omega), \nabla p(t, x) \in \vec{G}(\Omega)$.

Consider the orthogonal projection $P_{0}$ on $\vec{J}_{0}(\Omega)$ and the orthogonal projection $P_{G}$ on $\vec{G}(\Omega), P_{0}+P_{G}=I$. Assuming that $\vec{u}(t, x)$ and $\nabla p(t, x)$ are the classical solutions to problem (7.2) and applying the orthogonal projections $P_{0}$ and $P_{G}$ to both sides of the first equation in (7.2), we find

$$
\begin{gathered}
\frac{d \vec{u}}{d t}+\nu I_{0}(t)\left(A_{0} \vec{u}\right)=\vec{f}_{0}(t), \quad \vec{u}(0)=\vec{u}^{0}, \\
A_{0} \vec{u}:=-P_{0}(\Delta \vec{u}), \quad \vec{f}_{0}:=P_{0} \vec{f}, \\
\rho^{-1} \nabla p=\nu I_{0}(t) P_{G}(\Delta \vec{u})+P_{G} \vec{f} .
\end{gathered}
$$

Formula (7.8) shows that the pressure field $\nabla p(t, x)$ can be found provided that we know the velocity field $\vec{u}(t, x)$ regarded as a function of $t$ with values in $\vec{H}_{0}^{2} \cap$ $\vec{J}_{0}(\Omega)$, where $\vec{H}_{0}^{2}$ is the space of vector-valued functions with components in $H_{0}^{2}(\Omega)$. Therefore, it suffices to consider problem (7.6), where $\vec{u}=\vec{u}(t)$ is a function with values in $\vec{J}_{0}(\Omega)$ and $A_{0}$ is the Stokes operator, well known in hydrodynamics.

Problem (7.6) is a special case of the problem

$$
\frac{d u}{d t}+A_{0} u+\sum_{k=1}^{m} \int_{0}^{t} e^{-\gamma_{k}(t-s)} A_{k} u(s) d s=f(t), \quad u(0)=u^{0},
$$


where $u=u(t)$ is an unknown function with values in a separable Hilbert space $\mathcal{H}$, $\gamma_{k}$ are positive constants, $0<\gamma_{1}<\ldots<\gamma_{m}<\infty, f(t)$ is a given function with values in $\mathcal{H}, u^{0} \in \mathcal{H}$, and $A_{k}, k=0, \ldots, m$, are unbounded self-adjoint positive definite $\left(A_{k} \gg 0, k=0, \ldots, m\right)$ operators with domains $D\left(A_{k}\right), k=0, \ldots, m$, such that

$$
D\left(A_{k}\right)=D\left(A_{0}\right), \quad k=1, \ldots, m .
$$

For an example of such differential operators in the case $\mathcal{H}=L_{2}(\Omega), \Omega \subset \mathbb{R}^{n}$, we can take uniformly elliptic operators

$$
A_{k} u:=-\sum_{i, j=1}^{n} \frac{\partial}{\partial x_{i}}\left(a_{i j}^{(k)}(x) \frac{\partial u}{\partial x_{j}}\right), \quad k=0, \ldots, m,
$$

defined on the common domain

$$
D\left(A_{k}\right)=H_{0}^{2}(\Omega):=\left\{u(x) \in H^{2}(\Omega): u=0 \text { on } \partial \Omega\right\},
$$

where $H^{2}(\Omega)$ is the Sobolev space and $\partial \Omega \in C^{2}$.

Let $u(t)$ be a strong solution of problem (7.9). We introduce new unknown functions $u_{k}(t), k=0, \ldots, m$, by the formula

$$
u_{0}(t):=u(t), \quad u_{k}(t):=\int_{0}^{t} e^{-\gamma_{k}(t-s)} A_{k}^{1 / 2} u_{0}(s) d s, \quad k=1, \ldots, m .
$$

Then the equalities

$$
\begin{aligned}
\frac{d u_{k}}{d t} & =\frac{d}{d t} \int_{0}^{t} e^{-\gamma_{k}(t-s)} A_{k}^{1 / 2} u_{0}(s) d s=A_{k}^{1 / 2} u_{0}(t)-\gamma_{k} \int_{0}^{t} e^{-\gamma_{k}(t-s)} u_{0}(s) d s \\
& =A_{k}^{1 / 2} u_{0}(t)-\gamma_{k} u_{k}(t), \quad k=1, \ldots, m,
\end{aligned}
$$

together with (7.9) and (7.13) lead to the differential equation

$$
\frac{d \tilde{u}}{d t}+\mathcal{A}_{0} \tilde{u}=\tilde{f}(t), \quad \tilde{u}(0)=\tilde{u}^{0},
$$

in the Hilbert space

$$
\tilde{\mathcal{H}}:=\bigoplus_{k=0}^{m} \mathcal{H}_{k}, \quad \mathcal{H}_{k}:=\mathcal{H}, k=0,1, \ldots, m,
$$

where

$$
\begin{aligned}
\tilde{u}(t) & :=\left(u_{0}(t) ; \hat{u}_{1}(t)\right)^{T}, \quad \hat{u}_{1}(t):=\left(u_{1}(t) ; \ldots ; u_{m}(t)\right)^{T}, \\
\tilde{f}(t) & :=(f(t) ; \hat{0})^{T}, \quad \tilde{u}(0)=\left(u^{0} ; \hat{0}\right)^{T},
\end{aligned}
$$

and the operator $\mathcal{A}$ has the following matrix representation in the orthogonal decomposition (7.15):

$$
\begin{aligned}
& \mathcal{A}_{0}:=\left(A_{i j}\right)_{i, j=0}^{1}, \quad A_{00}:=A_{0}, \quad A_{01}:=\left(A_{1}^{1 / 2} ; \ldots ; A_{m}^{1 / 2}\right), \\
& A_{10}:=-\left(A_{1}^{1 / 2} ; \ldots ; A_{m}^{1 / 2}\right)^{T}, \quad A_{11}:=\operatorname{diag}\left(\gamma_{k} I\right)_{k=1}^{m} .
\end{aligned}
$$

Properties of the closure $\mathcal{A}$ of the operator $\mathcal{A}_{0}$ were studied in [1], in particular it was shown that the spectrum of the operator $\mathcal{A}$ is positive except for a finite number 
of nonreal eigenvalues counted with certain multiplicities. Thus, the operator $\mathcal{A}$ is of the $\left(f_{S}, f_{R}\right)$-type with

$$
f_{S}(\eta)=\gamma_{0} \cosh (a \eta), \quad f_{R}(z)= \begin{cases}1 /|\Im m z-s| & \text { if } \Re e z>\gamma_{0}, \\ \operatorname{dist}\left(z, s p\left(A_{a}\right)\right) & \text { otherwise }\end{cases}
$$

where $s$ is the half-width of the strip containing all eigenvalues.

As a third example of an operator with nonreal spectrum one can consider an analogous operator arising in the problem of small motions and normal oscillations of a viscous fluid in a partially filled container, where a problem of the type (7.14) can be obtained as the linearisation of the Navier-Stokes equations.

Finally, we note that square matrices with positive real part of the spectrum also belong to the class of operators of $\left(f_{S}, f_{R}\right)$-type.

\section{REFERENCES}

1. T.Ya. Azizov, N.D. Kopachevsky and L.D. Orlova: Evolution and spectral problems related to small motions of viscoelastic fluid. Amer. Math. Transl. (2) Vol 199, pp. 1-24, 2000. MR 2002b: 47102

2. M. Bebendorf and W. Hackbusch: Existence of $\mathcal{H}$-matrix approximants to the inverse FEmatrix of elliptic operators. Max-Planck-Institut für Mathematik in den Naturwissenschaften Leipzig, Preprint 21, 2002; Numer. Math. (to appear).

3. R. Bellman: Introduction to matrix analysis. Mc Graw-Hill Book Company, Inc., 1960. MR 23:A153

4. S. Börm, L. Grasedyck, and W. Hackbusch. Introduction to hierarchical matrices with applications. Max-Planck-Institut für Mathematik in den Naturwissenschaften Leipzig, Preprint Nr 18, 2002.

5. R. Dautray and J.-L. Lions: Mathematical analysis and numerical methods for science and technology, Vol. 5, evolutions problems I, Springer-Verlag 1992. MR 92k:00006

6. I.P. Gavrilyuk: Strongly P-positive operators and explicit representation of the solutions of initial value problems for second order differential equations in Banach space. J. Math. Anal. Appl. 236 (1999), 327-349. MR 2001j:34072

7. I.P. Gavrilyuk: An algorithmic representation of fractional powers of positive operators. Numer. Funct. Anal. Optim. 17 (1996), 293-305. MR 98b:47049

8. I.P. Gavrilyuk, W. Hackbusch and B.N. Khoromskij: $\mathcal{H}$-matrix approximation for the operator exponential with applications. Numer. Math. 92 (2002), 83-111.

9. I.P. Gavrilyuk,W. Hackbusch and B.N. Khoromskij: $\mathcal{H}$-matrix approximation for elliptic solution operators in cylinder domains. East-West J. Numer. Math. 9 (2001), 25-59. MR 2002e: 65064

10. I.P. Gavrilyuk and V.L. Makarov: Representation and approximation of the solution of an initial value problem for a first order differential equation in Banach space. Z. Anal. Anwend. 15 (1996), 495-527. MR 97h:65076

11. I.P. Gavrilyuk and V.L. Makarov: Explicit and approximate solutions of second order elliptic differential equations in Hilbert- and Banach spaces. Numer. Funct. Anal. Optim. 20 (1999), 695-717. MR 2001d:65157

12. I.P. Gavrilyuk and V.L. Makarov: Exponentially convergent parallel discretization methods for the first order evolution equations, Universität Leipzig, Preprint NTZ 12/2000.

13. L. Grasedyck, W. Hackbusch and B.N. Khoromskij: Application of $\mathcal{H}$-matrices in control theory. Max-Planck-Institut für Mathematik in den Naturwissenschaften Leipzig, Preprint 62, 2002; Computing 70 (2003), 121-165.

14. W. Hackbusch: A sparse matrix arithmetic based on $\mathcal{H}$-matrices. Part I: Introduction to $\mathcal{H}$-matrices. Computing 62 (1999), 89-108. MR 2000c:65039

15. W. Hackbusch and B. N. Khoromskij: A sparse $\mathcal{H}$-matrix arithmetic. Part II: Application to multi-dimensional problems. Computing 64 (2000), 21-47. MR 2001i:65053

16. W. Hackbusch and B. N. Khoromskij: A sparse $\mathcal{H}$-matrix arithmetic: General complexity estimates. J. Comp. Appl. Math. 125 (2000), 479-501. MR 2002f:65151 
17. M. Hochbruck and C. Lubich: On Krylov subspace approximations to the matrix exponential operator, SIAM J. Numer. Anal. 34 (1997), 1911-1925. MR 98h:65018

18. B.N. Khoromskij: Data-sparse elliptic operator inverse based on explicit approximation to the Green function, J. Numer. Math. 11, no. 2 (2003), 135-162.

19. Y. Maday, P. Pernaud-Thomas and H. Vandeven: Une réhabilitation des méthodes spectrales de type Laguerre, La Recherche Aérospatiale, Année 1985, No. 6 (Novembre-Décembre), pp. 353-375. MR 88b:65135

20. Y. Saad: Analysis of some Krylov subspace approximations to the matrix exponential operator, SIAM J. Numer. Anal. 29 (1992), 209-228. MR 92m:65050

21. D. Sheen, I. H. Sloan and V. Thomée: A parallel method for time-discretization of parabolic problems based on contour integral representation and quadrature, Math. Comp. 69 (2000), 177-195. MR 2000i:65161

22. F. Stenger: Numerical methods based on Sinc and analytic functions. Springer-Verlag, 1993. MR 94k:65003

23. F. Stenger: Collocating Convolutions, Math. Comp. 64 (1995), 211-235. MR 95c:65038

24. F. Stenger, B. Barkey and R. Vakili: in "Proceedings of Computation and Control III" (K. Bowers and J. Lund, eds.), Birkhäuser (1993), pp. 341-354.

Berufsakademie Thüringen, Am Wartenberg 2, D-99817 Eisenach, Germany

E-mail address: ipg@ba-eisenach.de

Max-Planck-Institute for Mathematics in the Sciences, Inselstr. 22-26, D-04103 LeIPZig, Germany

E-mail address: wh@mis.mpg.de

Max-Planck-Institute for Mathematics in the Sciences, Inselstr. 22-26, D-04103 LEIPZig, GERMAny

E-mail address: bokh@mis.mpg.de 